\title{
Classifying spectra of saturated fusion systems
}

\author{
KÁRI RAGNARSSON
}

The assignment of classifying spectra to saturated fusion systems was suggested by Linckelmann and Webb and has been carried out by Broto, Levi and Oliver. A more rigid (but equivalent) construction of the classifying spectra is given in this paper. It is shown that the assignment is functorial for fusion-preserving homomorphisms in a way which extends the assignment of stable $p$-completed classifying spaces to finite groups, and admits a transfer theory analogous to that for finite groups. Furthermore the group of homotopy classes of maps between classifying spectra is described, and in particular it is shown that a fusion system can be reconstructed from its classifying spectrum regarded as an object under the stable classifying space of the underlying p-group.

55R35; 20D20, 55P42

\section{Introduction}

Saturated fusion systems were introduced by Puig in $[20 ; 21]$ as a formalization of fusion systems of groups. To a finite group $G$ with Sylow $p$-subgroup $S$ one associates a fusion system $\mathcal{F}_{S}(G)$ over $S$. This is the category whose objects are the subgroups of $S$, and whose morphisms are the conjugations induced by elements in $G$. Puig axiomatized this construction, thus allowing abstract fusion systems without requiring the presence, or indeed existence, of an ambient group $G$. He also identified important properties enjoyed by those fusion systems that are induced by groups. Puig called fusion systems with these properties full Frobenius systems. These definitions were later simplified by Broto-Levi-Oliver, who introduced the term saturated fusion systems in [7] (see Definition 1.3 below). A further simplification has been obtained by Kessar-Stancu in [11].

A useful tool for the study of saturated fusion systems would be a functor assigning a classifying space to each saturated fusion system. Exactly what a classifying space means in this context is made precise by the theory of $p$-local finite groups developed by Broto-Levi-Oliver in [7]. They define a $p$-local finite group as a triple $(S, \mathcal{F}, \mathcal{L})$, where $S$ is a finite $p$-group, $\mathcal{F}$ is a saturated fusion system over $S$, and $\mathcal{L}$ is a centric 
linking system associated to $\mathcal{F}$, a category which contains just enough information to construct a classifying space $|\mathcal{L}|_{p}^{\wedge}$ for $\mathcal{F}$.

The motivating example for the definition of a $p$-local finite group comes from finite groups. In [6], Broto-Levi-Oliver give an algebraic construction for a centric linking system $\mathcal{L}_{S}^{c}(G)$ associated to the fusion system $\mathcal{F}_{S}(G)$ of a finite group $G$, and show that $\left|\mathcal{L}_{S}^{c}(G)\right|_{p}^{\wedge} \simeq B G_{p}^{\wedge}$.

Given the classifying space $|\mathcal{L}|_{p}^{\wedge}$, one can by [7] reconstruct the fusion system via the following homotopy-theoretic construction:

$$
\operatorname{Hom}_{\mathcal{F}}(P, Q)=\left\{\varphi \in \operatorname{Hom}(P, Q) \mid \theta \circ B \iota_{Q} \circ B \varphi \simeq \theta \circ B \iota_{P}\right\},
$$

where $\iota_{P}$ and $\iota_{Q} Q$ are the inclusions of the subgroups $P$ and $Q$ in $S$, and $\theta$ is the natural "inclusion" $B S \rightarrow|\mathcal{L}|_{p}^{\wedge}$. This construction was first applied by Martino-Priddy in [15] to show that if the $p$-completed classifying spaces of two finite groups have the same homotopy types, then their fusion systems are isomorphic.

The passage from saturated fusion systems to classifying spaces is more problematic. In general it is not known whether a saturated fusion system has an associated centric linking system, and if so, whether it is unique. Broto-Levi-Oliver have developed an obstruction theory to address these questions of existence and uniqueness. Oliver has shown in $[19 ; 18]$ that these obstructions vanish for fusion systems of finite groups. Therefore $\mathcal{L}_{S}^{c}(G)$ is, up to equivalence, the unique centric linking system associated to the fusion system $\mathcal{F}_{S}(G)$ of a finite group $G$. Moreover, Oliver concludes that the $p$-completed classifying spaces of two finite groups are homotopy equivalent if their fusion systems over chosen Sylow subgroups are isomorphic via a fusion-preserving isomorphism of these Sylow subgroups, thus proving the Martino-Priddy conjecture [15].

By Oliver's result the fusion system $\mathcal{F}_{S}(G)$ of a finite group $G$ has a unique associated centric linking system. But even when we restrict our attention to fusion systems coming from groups, we do not have an expedient method to reconstruct the linking system $\mathcal{L}_{S}^{c}(G)$ (and consequently $B G_{p}^{\wedge}$ ) from the fusion data $\mathcal{F}_{S}(G)$. Nor do we know whether this assignment is functorial, that is whether a morphism between fusion systems of groups induces a map between their $p$-completed classifying spaces.

A classifying space functor is not yet within our reach, but the stable analogue presents a more tractable problem. When calculating the cohomology of a $p$-local finite group $(S, \mathcal{F}, \mathcal{L})$ in [7], Broto-Levi-Oliver construct a characteristic biset for $\mathcal{F}$. This is an $(S, S)$-biset $\Omega$ with properties, suggested by Linckelmann-Webb, that guarantee that the induced stable selfmap of $B S$ is an idempotent in cohomology with $\mathbb{F}_{p}$-coefficients 
(see Proposition 4.2). Broto-Levi-Oliver noted that the stable summand of $\Sigma^{\infty} B S$ induced by a characteristic biset $\Omega$ is independent of the particular choice of $\Omega$, and agrees with the suspension spectrum of the classifying space $|\mathcal{L}|_{p}^{\wedge}$. Furthermore, they observed that the construction of $\Omega$ depends only on the saturated fusion system $\mathcal{F}$ and not on the centric linking system $\mathcal{L}$, and that therefore the induced summand $\mathbb{B} \mathcal{F}$ can be considered as a classifying spectrum for the saturated fusion system $\mathcal{F}$.

In this paper we take their idea further. We give a different formulation of the construction of a classifying spectrum of a saturated fusion system $\mathcal{F}$, which allows us to retain more information associated to $\mathcal{F}$. More precisely, we refine the construction of the biset $\Omega$ in [7] to produce an idempotent $\widetilde{\omega}$ in $\{B S, B S\}$ with the following stable idempotent analogues of the Linckelmann-Webb properties:

(a) $\widetilde{\omega}$ is a $\mathbb{Z}_{p}^{\wedge}$-linear combination of homotopy classes of maps of the form $\Sigma^{\infty} B \varphi \circ \operatorname{tr}_{P}$, where $P$ is a subgroup of $S, \varphi \in \operatorname{Hom}_{\mathcal{F}}(P, S)$ and $\operatorname{tr}_{P}$ denotes the reduced transfer of the inclusion $P \leq S$.

(b1) For each subgroup $P \leq S$ and each $\varphi \in \operatorname{Hom}_{\mathcal{F}}(P, S)$, the restrictions $\widetilde{\omega} \circ \Sigma^{\infty} B \iota_{P}$ and $\widetilde{\omega} \circ \Sigma^{\infty} B \varphi$ are homotopic as maps $\Sigma^{\infty} B P \rightarrow \Sigma^{\infty} B S$.

(b2) For each $P \leq S$ and each $\varphi \in \operatorname{Hom}_{\mathcal{F}}(P, S)$, the compositions $\operatorname{tr}_{P} \circ \tilde{\omega}$ and $t r_{\varphi} \circ \tilde{\omega}$, where $t r_{\varphi}$ is the reduced transfer of the monomorphism $\varphi: P \rightarrow S$, are homotopic as maps $\Sigma^{\infty} B S \rightarrow \Sigma^{\infty} B P$.

(c) $\tilde{\epsilon}(\omega)=1$, where $\tilde{\epsilon}:\{B S, B S\} \rightarrow \mathbb{Z}_{p}^{\wedge}$ is a morphism of modules derived from an augmentation of $\left\{B S_{+}, B S_{+}\right\}$(see Lemma 2.5 and Section 6).

We show that $\widetilde{\omega}$ is the unique idempotent in $\{B S, B S\}$ with these properties and that Property (b1) characterizes morphisms in the fusion system $\mathcal{F}$. Therefore we write $\widetilde{\omega}_{\mathcal{F}}$ and refer to $\widetilde{\omega}_{\mathcal{F}}$ as the stable characteristic idempotent of $\mathcal{F}$.

The homotopy type of the stable summand of $\Sigma^{\infty} B S$ induced by $\tilde{\omega}_{\mathcal{F}}$ agrees with the homotopy type of the classifying spectrum $\mathbb{B} \mathcal{F}$ constructed by Broto-Levi-Oliver, so this construction offers nothing new in itself. It is the careful study of the characteristic idempotent which allows us to exercise control over its mapping telescope $\mathbb{B} \mathcal{F}$. We refer to the structure map $\sigma_{\mathcal{F}}: \Sigma^{\infty} B S \rightarrow \mathbb{B} \mathcal{F}$ of the mapping telescope as the structure map of $\mathcal{F}$, and when regarded as an object under $\Sigma^{\infty} B S$, we refer to the pair $\left(\sigma_{\mathcal{F}}, \mathbb{B} \mathcal{F}\right)$ as the structured classifying spectrum of $\mathcal{F}$. The structure map $\sigma$ admits a transfer map $t_{\mathcal{F}}$, which is, up to homotopy, the unique map $\mathbb{B} \mathcal{F} \rightarrow B S$ such that $t_{\mathcal{F}} \circ \sigma_{\mathcal{F}} \simeq \widetilde{\omega}_{\mathcal{F}}$ and $\sigma_{\mathcal{F}} \circ t_{\mathcal{F}} \simeq i d_{\mathbb{B F}}$.

The reward for taking this point of view is the following result, which further justifies the use of the term "classifying spectrum". It appears in the text as Theorem 7.3. 
Theorem A If $\mathcal{F}$ is a saturated fusion system over a finite $p$-group $S$, then $\mathcal{F}$ can be recovered from its structured classifying spectrum $\left(\sigma_{\mathcal{F}}, \mathbb{B} \mathcal{F}\right)$ by the following homotopy-theoretic construction:

$$
\operatorname{Hom}_{\mathcal{F}}(P, Q)=\left\{\varphi \in \operatorname{Hom}(P, Q) \mid \sigma_{\mathcal{F}} \circ \Sigma^{\infty} B \iota_{Q} \circ \Sigma^{\infty} B \varphi \simeq \sigma_{\mathcal{F}} \circ \Sigma^{\infty} B \iota_{P}\right\},
$$

where $\iota_{P}$ and $\iota_{Q}$ are the inclusions of the subgroups $P$ and $Q$ in $S$.

By Martino-Priddy [14, Example 5.2] the fusion system can not be recovered from the homotopy type of the classifying spectrum alone; it must be regarded as an object under $\Sigma^{\infty} B S$. When applied to fusion systems of groups, this theorem gives an alternative stable classification of $p$-completed classifying spaces of finite groups, which is in some sense finer than the one in [14]. Combined with the Martino-Priddy conjecture, this shows that the unstable $p$-completed classifying space of a finite group is determined by the stable $p$-completed classifying space, regarded as an object under the stable classifying space of its $p$-Sylow subgroup. This matter is taken up in Ragnarsson [22].

The central result in this paper, which allows us to conduct the necessary analysis of characteristic idempotents, is the calculation of an explicit $\mathbb{Z}_{p}^{\wedge}$-basis for the submodule

$$
\widetilde{\omega}_{\mathcal{F}_{2}} \circ\left\{B S_{1}, B S_{2}\right\} \circ \widetilde{\omega}_{\mathcal{F}_{1}} \subset\left\{B S_{1}, B S_{2}\right\},
$$

for saturated fusion systems $\mathcal{F}_{1}$ and $\mathcal{F}_{2}$ over finite $p$-groups $S_{1}$ and $S_{2}$, respectively. This module is naturally isomorphic to the group of stable maps between the classifying spectra of the fusion systems involved and so we get the following theorem, a more concise version of which appears later as Theorem 7.2, as an immediate consequence.

Theorem B Let $\mathcal{F}_{1}$ and $\mathcal{F}_{2}$ be saturated fusion systems over finite $p$-groups $S_{1}$ and $S_{2}$, respectively. Then the group of homotopy classes of stable maps from $\mathbb{B} \mathcal{F}_{1}$ to $\mathbb{B} \mathcal{F}_{2}$ is a free $\mathbb{Z}_{p}^{\wedge}$-module with one basis element $\sigma_{\mathcal{F}_{2}} \circ\left(\Sigma^{\infty} B \psi \circ t r_{P}\right) \circ t_{\mathcal{F}_{1}}$ for every conjugacy class of pairs $(P, \psi)$ consisting of a subgroup $P \leq S_{1}$ and a nontrivial homomorphism $\psi: P \rightarrow S_{2}$. Conjugacy here means that $\mathcal{F}_{1}$-conjugacy is taken in the source and $\mathcal{F}_{2}$-conjugacy is taken in the target.

When $\mathcal{F}_{1}$ and $\mathcal{F}_{2}$ are fusion systems of groups, this theorem can be applied to give a new variant of the Segal conjecture describing the group of homotopy classes of stable maps between $p$-completed classifying spaces of finite groups. This discussion is taken up in Ragnarsson [24].

If $\mathcal{F}_{1}$ and $\mathcal{F}_{2}$ are saturated fusion systems over finite $p$-groups $S_{1}$ and $S_{2}$, respectively, the obstruction to restricting a homomorphism $\gamma: S_{1} \rightarrow S_{2}$ to a map between 
classifying spectra respecting their structure maps is the compatibility of $\gamma$ with the stable characteristic idempotents. This compatibility is achieved when $\gamma$ is a $\left(\mathcal{F}_{1}, \mathcal{F}_{2}\right)$-fusion-preserving homomorphism, which means that $\gamma$ induces a functor $F_{\gamma}: \mathcal{F}_{1} \rightarrow \mathcal{F}_{2}$ such that $F_{\gamma}(P)=\gamma(P)$ for all $P \leq S_{1}$ and $\left.\gamma\right|_{Q} \circ \varphi=\left.F_{\gamma}(\varphi) \circ \gamma\right|_{P}$ for all $\varphi \in \operatorname{Hom}_{\mathcal{F}_{1}}(P, Q)$. Letting SFS denote the category whose objects are the saturated fusion systems and whose morphisms are fusion-preserving homomorphisms, we get the following result which follows from Theorem 7.9 in the text.

Theorem C There is a classifying spectrum functor

$$
\mathbb{B}: \mathrm{SFS} \longrightarrow \text { Spectra }
$$

acting on objects by sending a saturated fusion system to its classifying spectrum and on morphisms by sending a $\left(\mathcal{F}_{1}, \mathcal{F}_{2}\right)$-fusion-preserving morphism $\gamma$ to the map

$$
\mathbb{B} \gamma_{\mathcal{F}_{1}}^{\mathcal{F}_{2}}:=\sigma_{\mathcal{F}_{2}} \circ \Sigma^{\infty} B \gamma \circ t_{\mathcal{F}_{1}}: \mathbb{B} \mathcal{F}_{1} \longrightarrow \mathbb{B} \mathcal{F}_{2},
$$

which satisfies

$$
\mathbb{B} \gamma_{\mathcal{F}_{1}}^{\mathcal{F}_{2}} \circ \sigma_{\mathcal{F}_{1}} \simeq \sigma_{\mathcal{F}_{2}} \circ \Sigma^{\infty} B \gamma
$$

It is an important property of this functor that when $\gamma: S_{1} \rightarrow S_{2}$ is the restriction of a homomorphism $\bar{\gamma}: G_{1} \rightarrow G_{2}$ to Sylow subgroups, the map $\mathbb{B} \mathcal{F}_{S_{1}}\left(G_{1}\right) \rightarrow \mathbb{B} \mathcal{F}_{S_{2}}\left(G_{2}\right)$ induced by $\gamma$ is equivalent to the map $\Sigma^{\infty} B G_{1} \hat{p} \rightarrow \Sigma^{\infty} B G_{2} \hat{p}$ induced by $\bar{\gamma}$, as maps of objects under the stable classifying spaces of their Sylow subgroups. This is proved in Section 10.

A monomorphism $\gamma: S_{1} \rightarrow S_{2}$ admits a transfer map $t r_{\gamma}: \Sigma^{\infty} B S_{2} \rightarrow \Sigma^{\infty} B S_{1}$, which restricts to a map of classifying spectra that preserves transfer maps when $\gamma$ is fusion-preserving. Collecting Theorem 8.6 and Propositions 9.5 and 9.6, we get the following result.

Theorem D There is an assignment of a transfer map

$$
\operatorname{Tr}\left(\gamma_{\mathcal{F}_{1}}^{\mathcal{F}_{2}}\right):=\sigma_{\mathcal{F}_{1}} \circ t r_{\gamma} \circ t_{\mathcal{F}_{2}}: \mathbb{B} \mathcal{F}_{2} \rightarrow \mathbb{B} \mathcal{F}_{1},
$$

to every $\left(\mathcal{F}_{1}, \mathcal{F}_{2}\right)$-fusion-preserving monomorphism $\gamma: S_{1} \rightarrow S_{2}$. The assignment has the following properties:

(i) $t_{\mathcal{F}_{1}} \circ \operatorname{Tr}\left(\gamma_{\mathcal{F}_{1}}^{\mathcal{F}_{2}}\right) \simeq t r_{\gamma} \circ t_{\mathcal{F}_{2}}$.

(ii) $\operatorname{Tr}\left(\gamma_{\mathcal{F}_{1}}^{\mathcal{F}_{2}}\right) \circ \operatorname{Tr}\left(\gamma_{\mathcal{F}_{2}}^{\mathcal{F}_{3}}\right) \simeq \operatorname{Tr}\left(\gamma_{\mathcal{F}_{1}}^{\mathcal{F}_{3}}\right)$.

(iii) The composition $\mathbb{B} \gamma_{\mathcal{F}_{1}}^{\mathcal{F}_{2}} \circ \operatorname{Tr}\left(\gamma_{\mathcal{F}_{1}}^{\mathcal{F}_{2}}\right)$ acts on $H^{*}\left(\mathbb{B} \mathcal{F}_{2} ; \mathbb{F}_{p}\right)$ as multiplication by $\left|S_{2}\right| /\left|S_{1}\right|$. 
(iv) The transfer $\operatorname{Tr}\left(\gamma_{\mathcal{F}_{1}}^{\mathcal{F}_{2}}\right)$ satisfies the Frobenius reciprocity relation

$$
\begin{gathered}
\operatorname{Tr}\left(\gamma_{\mathcal{F}_{1}}^{\mathcal{F}_{2}}\right)^{*}\left(\mathbb{B} \gamma_{\mathcal{F}_{1}}^{\mathcal{F}_{2}{ }^{*}}(x) \cdot y\right)=x \cdot \operatorname{Tr}\left(\gamma_{\mathcal{F}_{1}}^{\mathcal{F}_{2}}\right)^{*}(y) \\
\text { for } x \in H^{*}\left(\mathbb{B} \mathcal{F}_{2} ; \mathbb{F}_{p}\right) \text { and } y \in H^{*}\left(\mathbb{B} \mathcal{F}_{1} ; \mathbb{F}_{p}\right) .
\end{gathered}
$$

The motivation for the work in this paper comes from a question of Miller regarding an alternative formulation of $p$-local finite groups in terms of homotopy subgroup inclusions satisfying a certain transfer property. The author has obtained a partial answer to this question, but on the way to doing so, has discovered results about classifying spectra of saturated fusion systems which are most likely of interest to a wider audience than the original question, and are therefore presented separately in this paper. These results do not depend on centric linking systems, and to emphasize this we mostly avoid mentioning centric linking systems in this paper. Implications for $p$-local finite groups will be discussed in a subsequent paper [23], where Miller's question will be addressed.

Notational conventions Throughout this paper, $p$ is a fixed prime. Cohomology is always taken with $\mathbb{F}_{p}$-coefficients. For a space $X$ we let $X_{+}$be the pointed space obtained by adding a disjoint basepoint to $X$, and we let $X_{p}^{\wedge}$ denote the Bousfield-Kan p-completion [5].

The category of finite groups and homomorphisms is denoted by Gr. For an element $g$ of a group $G$, we let $c_{g}$ denote the conjugation $x \mapsto g x g^{-1}$. When $H$ is a subgroup of $G$ we write ${ }^{g} H$ for the conjugate $c_{g}(H)$ and $H^{g}$ for the inverse conjugate $c_{g}^{-1}(H)=g^{-1} H g$. For subgroups $H$ and $K$ of $G$ we let $N_{G}(H, K)$ denote the transporter

$$
N_{G}(H, K):=\left\{g \in G \mid{ }^{h} H \leq K\right\},
$$

and write

$$
\begin{aligned}
\operatorname{Hom}_{G}(H, K): & =\left\{c_{g}: H \rightarrow K \mid g \in N_{G}(H, K)\right\} \\
& =N_{G}(H, K) / C_{G}(H)
\end{aligned}
$$

for the set of homomorphisms from $H$ to $K$ induced by conjugation in $G$.

The inclusion of a subgroup $H$ into a supergroup is denoted by $\iota_{H}$, specifying the supergroup when there is danger of confusion. For the convenience of the reader we use the letters $S, P$ and $Q$ to refer to finite $p$-groups, while $G$ and $H$ refer to general finite groups. Moreover we use $\varphi$ to denote homomorphisms belonging to fusion systems, while $\psi$ and $\rho$ denote general homomorphisms. 
All stable homotopy takes place in the homotopy category of spectra which we denote by Spectra. A discussion of the stable homotopy category can be found for example in [1]. We will use the shorthand notation

$$
\Sigma_{+}^{\infty} X:=\Sigma^{\infty}\left(X_{+}\right)
$$

for the suspension spectrum of $X_{+}$. Since we often have cause to work with stable $p$-completed classifying spaces, we adopt the shorthand notation

$$
\mathbb{B}(-):=\Sigma^{\infty} B(-)_{p}^{\wedge},
$$

regarded as functors

$$
\mathrm{Gr} \longrightarrow \text { Spectra. }
$$

As is usual, for spaces $X$ and $Y$ we let $\{X, Y\}$ denote the group of homotopy classes of stable maps $\Sigma^{\infty} X \rightarrow \Sigma^{\infty} Y$, and for spectra $E$ and $F$ we let $[E, F]$ denote the group of homotopy classes of (degree 0 ) maps $E \rightarrow F$. All homotopies are unpointed.

Overview In the first section we recall the definition of saturated fusion systems. The second section treats Burnside modules and the Segal conjecture relating them to stable maps between classifying spaces of groups. In addition we develop some tools and notation we will use throughout the paper. In Section 3 we introduce the notion of fusion subconjugacy. For fusion systems $\mathcal{F}_{1}$ and $\mathcal{F}_{2}$ over finite $p$-groups $S_{1}$ and $S_{2}$, this gives a useful fusion-invariant filtration of the Burnside module $A\left(S_{1}, S_{2}\right)$. In Section 4 we assign a characteristic idempotent $\omega_{\mathcal{F}}$ in the $p$-completed double Burnside ring $A(S, S)_{p}^{\wedge}$ to a saturated fusion system $\mathcal{F}$ over $S$. In Section 5 we perform a careful analysis of the inherent properties of this idempotent, and in Section 6 we interpret these results for the stable idempotent $\tilde{\omega}_{\mathcal{F}}$ of $\mathbb{B} S$ induced by $\omega_{\mathcal{F}}$. In Section 7 we define the classifying spectrum of $\mathcal{F}$ as the summand $\mathbb{B} \mathcal{F}$ of $\Sigma^{\infty} B S$ given by $\tilde{\omega}_{\mathcal{F}}$, and prove that this assignment is functorial. In Section 8 we develop the theory of transfers for classifying spectra, and in Section 9 we look at the behaviour of classifying spectra and their transfers in cohomology. We conclude this paper in Section 10 by showing that the theory of classifying spectra of saturated fusion systems developed here agrees with existing theories of stable classifying spaces of saturated fusion systems.

Acknowledgements I would like to thank my thesis advisor Haynes Miller for suggesting the problem out of which this work grew and for his enthusiasm and helpful advice during its progress. I also thank Bob Oliver for his emailed suggestions for the proof of convergence in Section 4 and Ran Levi for many lively and encouraging discussions on this subject. Finally I thank the referee on behalf of both the reader and 
myself for a very accurate and helpful report which has improved the exposition and clarity of the paper. The method of proof of the central result in this paper is, at least subconsciously, inspired by Nishida's work in [17] and a preliminary version thereof.

The author was supported by EPSRC grant GR/S94667/01 during part of this work.

\section{Saturated fusion systems}

In this section we recall the definition of a saturated fusion system. We begin by presenting the motivating example.

Definition 1.1 Let $G$ be a finite group with Sylow $p$-subgroup $S$. The fusion system of $G$ over $S$ is the category $\mathcal{F}_{S}(G)$ whose objects are the subgroups of $S$, and whose morphisms are the homomorphisms induced by conjugation in $G$ :

$$
\operatorname{Hom}_{\mathcal{F}_{S}(G)}(P, Q)=\operatorname{Hom}_{G}(P, Q) .
$$

Puig $[20 ; 21]$ axiomatized this construction as follows.

Definition 1.2 A fusion system $\mathcal{F}$ over a finite $p$-group $S$ is a category, whose objects are the subgroups of $S$, and whose morphism sets $\operatorname{Hom}_{\mathcal{F}}(P, Q)$ satisfy the following conditions:

(a) $\operatorname{Hom}_{S}(P, Q) \subseteq \operatorname{Hom}_{\mathcal{F}}(P, Q) \subseteq \operatorname{Inj}(P, Q)$ for all $P, Q \leq S$.

(b) Every morphism in $\mathcal{F}$ factors as an isomorphism in $\mathcal{F}$ followed by an inclusion.

From the definition it is clear that every fusion system over $S$ contains the fusion system $\mathcal{F}_{S}(S)$ of $S$. We denote this fusion system by $\mathcal{F}_{S}$ for short.

Fusion systems at this level of generality are not particularly useful or interesting, so we restrict to a certain subclass of fusion systems introduced by Puig in [20]. Puig identified important properties enjoyed by fusion systems of groups, and called fusion systems with these properties full Frobenius systems. His definitions were later simplified by Broto-Levi-Oliver in [7], where they suggested the name saturated fusion systems. A further simplification has been obtained by Kessar-Stancu in [11].

We present the Broto-Levi-Oliver version below, but before stating the definition, we need to introduce some additional terminology. We say that two subgroups $P, P^{\prime} \leq S$ are $\mathcal{F}$-conjugate if they are isomorphic in $\mathcal{F}$. A subgroup $P \leq S$ is fully centralized in $\mathcal{F}$ if $\left|C_{S}(P)\right| \geq\left|C_{S}\left(P^{\prime}\right)\right|$ for every $P^{\prime} \leq S$ that is $\mathcal{F}$-conjugate to $P$. Similarly $P$ is fully normalized in $\mathcal{F}$ if $\left|N_{S}(P)\right| \geq\left|N_{S}\left(P^{\prime}\right)\right|$ for every $P^{\prime} \leq S$ that is $\mathcal{F}$-conjugate to $P$. 
Definition 1.3 A fusion system $\mathcal{F}$ over a $p$-group $S$ is saturated if the following two conditions hold:

(I) If $P \leq S$ is fully normalized in $\mathcal{F}$, then $P$ is also fully centralized in $\mathcal{F}$, and $p$ does not divide the index of $\operatorname{Aut}_{S}(P)$ in $\operatorname{Aut}_{\mathcal{F}}(P)$.

(II) If $P \leq S$ and $\varphi \in \operatorname{Hom}_{\mathcal{F}}(P, S)$ are such that $\varphi(P)$ is fully centralized, then $\varphi$ extends to $\bar{\varphi} \in \operatorname{Hom}_{\mathcal{F}}\left(N_{\varphi}, S\right)$, where

$$
N_{\varphi}=\left\{g \in N_{S}(P) \mid \varphi \circ c_{g} \circ \varphi^{-1} \in \operatorname{Aut}_{S}(\varphi(P))\right\} .
$$

This definition is rather technical, and as the conditions in the definition are not used explicitly in this paper, it suffices for the reader to keep in mind that Condition I is a "prime to $p$ " or "Sylow" property, analogous to the fact that the index of a Sylow subgroup in a finite group is not divisible by $p$. Condition II is a "maximal extension property" which (in a non-precise sense and when combined with Condition I) can be thought of as an axiomatic replacement of Sylow's Second and Third Theorems.

The role of saturated fusion systems in the theory of classifying spectra developed in this paper is as follows. In Section 4 we construct a characteristic idempotent $\omega$ for a fusion system $\mathcal{F}$ with a characteristic biset $\Omega$. These objects are defined precisely in Section 4 , and for now it suffices to say that characteristic bisets are finite $(S, S)$ bisets with properties stipulated by Linckelmann-Webb. The classifying spectrum of $\mathcal{F}$ is then constructed using $\omega$ in Section 7. A construction of characteristic bisets for saturated fusion systems is given by Broto-Levi-Oliver in [7]. This allows us to develop the theory of classifying spectra of saturated fusion system. But existence of a classifying spectrum for a fusion system $\mathcal{F}$ depends only on the existence of a characteristic biset for $\mathcal{F}$, and the properties of classifying spectra follow from the Linckelmann-Webb properties without using the saturation axioms. The theory therefore extends automatically to all fusion systems that have characteristic bisets. It is an interesting question whether the existence of a characteristic biset for a fusion system $\mathcal{F}$ implies saturation of $\mathcal{F}$. The author believes this is true, which is why the results in this paper are only presented for saturated fusion systems.

\section{Burnside modules and the Segal conjecture}

In this section we give a brief discourse about how stable maps between classifying spaces of finite groups $G_{1}$ and $G_{2}$ are related to $\left(G_{1}, G_{2}\right)$-bisets.

For finite groups $G_{1}$ and $G_{2}$, let $A^{+}\left(G_{1}, G_{2}\right)$ be the set of isomorphism classes of finite sets with a right $G_{1}$-action and a free left $G_{2}$-action. The disjoint union operation 
makes $A^{+}\left(G_{1}, G_{2}\right)$ into a commutative monoid. We denote the Grothendieck group completion by $A\left(G_{1}, G_{2}\right)$ and refer to it as the Burnside module of $G_{1}$ and $G_{2}$. The reader should beware that this is not standard terminology. The group structure of $A\left(G_{1}, G_{2}\right)$ is easy to describe. It is a free abelian group with one generator corresponding to each transitive $\left(G_{1}, G_{2}\right)$-biset. We proceed to describe and parametrize these basis elements below.

Definition 2.1 Let $G_{1}$ and $G_{2}$ be finite groups. A $\left(G_{1}, G_{2}\right)$-pair is a pair $(H, \psi)$ consisting of a subgroup $H \leq G_{1}$ and a homomorphism

$$
\psi: H \rightarrow G_{2} .
$$

We say that two $\left(G_{1}, G_{2}\right)$-pairs $\left(H_{1}, \psi_{1}\right)$ and $\left(H_{2}, \psi_{2}\right)$ are $\left(G_{1}, G_{2}\right)$-conjugate if there exist elements $g \in G_{1}$ and $h \in G_{2}$ such that $c_{g}\left(H_{1}\right)=H_{2}$ and the following diagram commutes

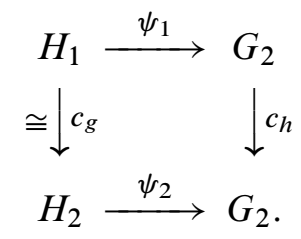

Remark 2.2 Define the graph of a $\left(G_{1}, G_{2}\right)$-pair $(H, \psi)$ by

$$
\Delta_{H}^{\psi}:=\left\{(h, \psi(h) \mid h \in H\} \leq G_{1} \times G_{2} .\right.
$$

It is easy to check that $\left(G_{1}, G_{2}\right)$-pairs $\left(H_{1}, \psi_{1}\right)$ and $\left(H_{2}, \psi_{2}\right)$ are $\left(G_{1}, G_{2}\right)$-conjugate if and only if their graphs are conjugate in $G_{1} \times G_{2}$.

We denote the $\left(G_{1}, G_{2}\right)$-conjugacy class of a $\left(G_{1}, G_{2}\right)$-pair $(H, \psi)$ by $[H, \psi]_{G_{1}}^{G_{2}}$ or, when there is no danger of confusion, just $[H, \psi]$. With a slight abuse of notation we will also let $[H, \psi]_{G_{1}}^{G_{2}}$ (or $[H, \psi]$ ) denote the basis element of $A\left(G_{1}, G_{2}\right)$ corresponding to the conjugacy class of the $\left(G_{1}, G_{2}\right)$-pair $(H, \psi)$. Thus $[H, \psi]$ represents the isomorphism class of the $\left(G_{1}, G_{2}\right)$-biset

$$
G_{2} \times(H, \psi) G_{1}:=\left(G_{2} \times G_{1}\right) / \sim,
$$

with the obvious right $G_{1}$-action and left $G_{2}$-action, where

$$
(x, g y) \sim(x \psi(g), y)
$$

for $x \in G_{2}, y \in G_{1}$ and $g \in H$.

Given three finite groups $G_{1}, G_{2}$, and $G_{3}$, we get a morphism of monoids

$$
\text { - ०-: } A^{+}\left(G_{2}, G_{3}\right) \times A^{+}\left(G_{1}, G_{2}\right) \rightarrow A^{+}\left(G_{1}, G_{3}\right)
$$


by

$$
(\Omega, \Lambda) \mapsto \Omega \circ \Lambda:=\Omega \times_{G_{2}} \Lambda,
$$

which extends to a bilinear map

$$
A\left(G_{2}, G_{3}\right) \times A\left(G_{1}, G_{2}\right) \rightarrow A\left(G_{1}, G_{3}\right) .
$$

This pairing can be described in terms of the basis elements using the double coset formula.

$$
[K, \rho]_{G_{2}}^{G_{3}} \circ[H, \psi]_{G_{1}}^{G_{2}}=\sum_{x \in K \backslash G_{2} / \psi(H)}\left[\psi^{-1}\left(\psi(H) \cap K^{x}\right), \rho \circ c_{x} \circ \psi\right]_{G_{1}}^{G_{3}} .
$$

We pay special attention to the simple case where $K=G_{2}$, so $\rho$ and $\psi$ are composable morphisms. In this case the double coset formula simplifies to

$$
\left[G_{2}, \rho\right]_{G_{2}}^{G_{3}} \circ[H, \psi]_{G_{1}}^{G_{2}}=[H, \rho \circ \psi]_{G_{1}}^{G_{3}} .
$$

For a finite group $G$ the pairing of (1) makes $A(G, G)$ into a ring which we call the double Burnside ring of $G$. This should not be confused with the Burnside ring $A(G)$ [25]. The latter is the Grothendieck group completion of the monoid of isomorphism classes of finite left $G$-sets. As a $\mathbb{Z}$-module, $A(G)$ is free $\mathbb{Z}$-module with one generator $[G / H]$ for each conjugacy class of subgroups $H \leq G$. As a ring, the multiplicative structure on $A(G)$ is induced by Cartesian product and linear extension.

Bisets relate to stable maps via the Becker-Gottlieb transfer [3]. We recall some basic properties of transfers here, and refer the reader to [2] for a more thorough discussion. Given a finite covering $f: X \rightarrow Y$, where $Y$ is connected, Becker-Gottlieb constructed a stable map $t r_{f}: \Sigma_{+}^{\infty} Y \rightarrow \Sigma_{+}^{\infty} X$, called the transfer of $f$. (Actually, a more general transfer for fibrations with compact fibres has been constructed by Dwyer in [10] but we need not consider that here.) We will use the following important properties of transfers:

Contravariant functoriality If $f: X \rightarrow Y$ and $g: Y \rightarrow Z$ are finite coverings of connected spaces, then

$$
t r_{g \circ f} \simeq t r_{f} \circ t r_{g}
$$

Normalization If $f: X \rightarrow Y$ is an $n$-fold cover of a connected space, then the induced map in singular cohomology (with any coefficients)

$$
t r_{f}^{*} \circ \Sigma_{+}^{\infty} f^{*}: H^{*}(Y) \rightarrow H^{*}(X) \rightarrow H^{*}(Y)
$$

is multiplication by $n$. 
Frobenius reciprocity If $f: X \rightarrow Y$ is a finite cover of a connected space, then the following diagram, where $\Delta_{X}$ and $\Delta_{Y}$ denote the respective diagonals of $X$ and $Y$, commutes:

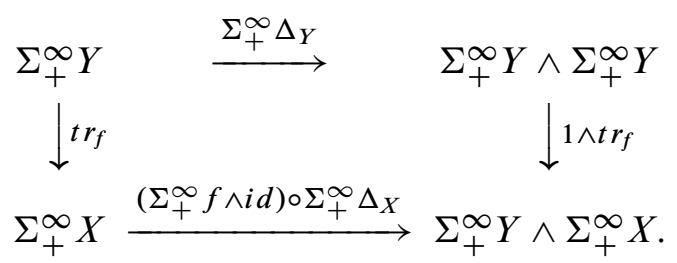

In particular,

$$
\operatorname{tr}_{f}^{*}\left(f^{*}(y) \cdot x\right)=y \cdot \operatorname{tr}_{f}^{*}(x)
$$

for $x \in H^{*}(X)$ and $y \in H^{*}(Y)$.

Since $\Sigma_{+}^{\infty} X \simeq \Sigma^{\infty} X \vee \mathbb{S}^{0}$, the transfer $\operatorname{tr}_{f}: \Sigma_{+}^{\infty} Y \rightarrow \Sigma_{+}^{\infty} X$ of a finite cover $f: X \rightarrow Y$ restricts to a reduced transfer $\Sigma^{\infty} X \rightarrow \Sigma^{\infty} Y$. As there is no danger of confusion we also denote the reduced transfer by $t r_{f}$, and sometimes refer to it as transfer.

A monomorphism of groups $\psi: G \rightarrow G^{\prime}$ induces a fibration $G^{\prime} / \psi(G) \hookrightarrow B G \stackrel{B \psi}{\longrightarrow}$ $B G^{\prime}$. If $\left[G^{\prime}: \psi(G)\right]$ is finite, which is always the case if $G$ and $G^{\prime}$ are finite, $B \psi$ therefore admits a transfer map, which we denote $t r_{\psi}$ for short. In the special case of the inclusion $H \leq G$ of a subgroup of finite index we denote the transfer by $t r_{H}$.

Given a finite $\left(G_{1}, G_{2}\right)$-biset $\Omega \in A^{+}\left(G_{1}, G_{2}\right)$, we now get a stable map $\alpha(\Omega) \in\left\{B G_{1+}, B G_{2+}\right\}$ as follows. Let $\Lambda:=G_{2} \backslash \Omega$. Since the left $G_{2}$-action on $\Omega$ is free, we get a principal fibre sequence

$$
G_{2} \rightarrow \Omega \times_{G_{1}} E G_{1} \rightarrow \Lambda \times_{G_{1}} E G_{1} .
$$

Let

$$
\xi: \Lambda \times_{G_{1}} E G_{1} \rightarrow B G_{2}
$$

be the classifying map of this fibration. The projection map

$$
\Lambda \times_{G_{1}} E G_{1} \rightarrow B G_{1}
$$

is a finite covering. Let $\tau: \Sigma_{+}^{\infty} B G_{1} \rightarrow \Sigma_{+}^{\infty}\left(\Lambda \times_{G_{1}} E G_{1}\right)$ be the associated transfer map. The map $\alpha(\Omega)$ is now defined as

$$
\alpha(\Omega):=\Sigma_{+}^{\infty} \xi \circ \tau .
$$

This assignment extends to a homomorphism

$$
\alpha: A\left(G_{1}, G_{2}\right) \rightarrow\left\{B G_{1+}, B G_{2+}\right\}
$$

Algebraic 83 Geometric Topology, Volume 6 (2006) 
of abelian groups. Although it may not be immediate from the definition, it is shown for example in [4] that the map $\alpha$ is natural in the sense that it sends the pairing of (1) to the composition pairing for stable maps:

$$
\alpha(\Omega \circ \Lambda)=\alpha(\Omega) \circ \alpha(\Lambda) .
$$

Thus $\alpha$ is a ring homomorphism when $G_{1}=G_{2}$. One can check that the value of $\alpha$ on a basis element $[H, \psi]$ is

$$
\alpha([H, \psi])=\Sigma_{+}^{\infty} B \psi \circ \operatorname{tr}_{H} .
$$

The homomorphism $\alpha$ gives a way to relate $A\left(G_{1}, G_{2}\right)$ to the group of homotopy classes of stable maps $\left\{B G_{1+}, B G_{2+}\right\}$. Lewis-May-McClure have made this relationship precise in [12]. As a consequence of the Segal conjecture (proved by Carlsson in [8]), they show that $\alpha$ is completion with respect to the augmentation ideal $I\left(G_{1}\right)$ of the Burnside ring $A\left(G_{1}\right)$. In the case where $G_{1}$ is a $p$-group, May-McClure [16] showed that, after getting rid of basepoints, this completion takes a simple form, which we will describe below.

Definition 2.3 For finite groups $G_{1}$ and $G_{2}$, we say that a $\left(G_{1}, G_{2}\right)$-pair $(H, \psi)$ is trivial if $\psi$ is the trivial homomorphism. In this case we also say that the conjugacy class $[H, \psi]$ is trivial. When $\psi$ is not the trivial homomorphism, we say that the pair $(H, \psi)$ and the conjugacy class $[H, \psi]$ are non-trivial.

Let $\widetilde{A}\left(G_{1}, G_{2}\right)$ be the quotient module obtained from $A\left(G_{1}, G_{2}\right)$ by quotienting out all trivial basis elements $[H, \psi]$. Recalling that $\Sigma_{+}^{\infty} B G \simeq \Sigma^{\infty} B G \vee \mathbb{S}^{0}$, where $\mathbb{S}^{0}=\Sigma^{\infty} S^{0}$ is the suspension sphere spectrum, one can check that there is an induced map

$$
\alpha: \tilde{A}\left(G_{1}, G_{2}\right) \longrightarrow\left\{B G_{1+}, B G_{2+}\right\} /\left\{B G_{1+}, S^{0}\right\} \cong\left\{B G_{1}, B G_{2}\right\} .
$$

May-McClure proved that when $G_{1}$ is a $p$-group, $I\left(G_{1}\right)$-adic completion coincides with $p$-adic completion on $\widetilde{A}\left(G_{1}, G_{2}\right)$, and deduced the following version of the Segal conjecture.

Theorem 2.4 (Segal conjecture $[8 ; 12 ; 16]$ ) If $S$ is a finite $p$-group and $G$ any finite group, then $\alpha$ induces an isomorphism

$$
\tilde{\alpha}: \tilde{A}(S, G)_{p}^{\wedge} \cong\{B S, B G\}
$$

where $(-)_{p}^{\wedge}=(-) \otimes \mathbb{Z}_{p}^{\wedge}$ is $p$-adic completion. 
For finite $p$-groups $S_{1}$ and $S_{2}$, we will, in view of the Segal conjecture, have reason to $p$-complete the Burnside module $A\left(S_{1}, S_{2}\right)$. The resulting $\mathbb{Z}_{p}^{\wedge}$-module $A\left(S_{1}, S_{2}\right)_{p}^{\wedge}$ is a free $\mathbb{Z}_{p}^{\wedge}$-module with one basis element for each conjugacy class of $\left(S_{1}, S_{2}\right)$-pairs, and by a further, yet slight, abuse of notation, we will also let $[P, \psi]_{S_{1}}^{S_{2}}$ (or $[P, \psi]$ ) denote the basis element of $A\left(S_{1}, S_{2}\right)_{p}^{\wedge}$ corresponding to the conjugacy class of the $\left(S_{1}, S_{2}\right)$-pair $(P, \psi)$.

We conclude this section by adapting some "bookkeeping" tools for $\left(S_{1}, S_{2}\right)$-bisets to keep track of elements of $A\left(S_{1}, S_{2}\right)_{p}^{\wedge}$. First we note that the structure of $A\left(S_{1}, S_{2}\right)_{p}^{\wedge}$ allows us to define a collection of homomorphisms

$$
\chi_{[P, \psi]}: A\left(S_{1}, S_{2}\right)_{p}^{\wedge} \rightarrow \mathbb{Z}_{p}^{\wedge},
$$

one for each conjugacy class of $\left(S_{1}, S_{2}\right)$-pairs, by demanding that

$$
\Omega=\sum_{[P, \psi]} \chi_{[P, \psi]}(\Omega) \cdot[P, \psi]
$$

for all $\Omega \in A\left(S_{1}, S_{2}\right)_{p}^{\wedge}$.

Next, we extend the notion of counting the number of $S_{2}$-orbits of $\left(S_{1}, S_{2}\right)$-bisets to obtain a form of augmentation for Burnside modules. The resulting assignment is natural in that it sends the pairing of (1) to multiplication in $\mathbb{Z}_{p}^{\wedge}$.

Lemma 2.5 For every pair of finite $p$-groups $S_{1}$ and $S_{2}$, the assignment

$$
A^{+}\left(S_{1}, S_{2}\right) \rightarrow \mathbb{Z}, \quad \Omega \mapsto\left|S_{2} \backslash \Omega\right|
$$

extends to a homomorphism

$$
\epsilon: A\left(S_{1}, S_{2}\right)_{p}^{\wedge} \rightarrow \mathbb{Z}_{p}^{\wedge},
$$

sending composition to multiplication.

Proof Recalling that bisets $\Omega \in A^{+}\left(S_{1}, S_{2}\right)$ have a free $S_{2}$-action, we see that each assignment

$$
A^{+}\left(S_{1}, S_{2}\right) \rightarrow \mathbb{Z}, \quad \Omega \mapsto\left|S_{2} \backslash \Omega\right|
$$

is a morphism of monoids, and so we get an induced homomorphism $\epsilon: A\left(S_{1}, S_{2}\right) \rightarrow \mathbb{Z}$ and, after $p$-completion, an induced homomorphism $\epsilon: A\left(S_{1}, S_{2}\right)_{p}^{\wedge} \rightarrow \mathbb{Z}_{p}^{\wedge}$.

Using the freeness of the left action for bisets $\Omega \in A^{+}\left(S_{2}, S_{3}\right)$ and $\Lambda \in A^{+}\left(S_{1}, S_{2}\right)$ again, we get

$$
\left|S_{3} \backslash(\Omega \circ \Lambda)\right|=\left|\Omega \times_{S_{2}} \Lambda\right| /\left|S_{3}\right|=\left(|\Omega| \cdot|\Lambda| /\left|S_{2}\right|\right) /\left|S_{3}\right|=\left|S_{3} \backslash \Omega\right| \cdot\left|S_{2} \backslash \Lambda\right| .
$$


The collection of homomorphisms $\epsilon: A\left(S_{1}, S_{2}\right)_{p}^{\wedge} \rightarrow \mathbb{Z}_{p}^{\wedge}$ therefore sends composition to multiplication.

A useful, well known result states that for a finite group $G$, two finite $G$-sets $\Omega$ and $\Lambda$ are isomorphic if and only if they have the same number of fixed points for every subgroup of $G$. Since the number of fixed points depends only on the conjugacy class of the subgroup, an alternative formulation is that there is an injective $\mathbb{Z}$-module homomorphism

$$
A(G) \rightarrow \prod_{[H]} \mathbb{Z}, \quad \Omega \mapsto \prod_{[H]}\left|\Omega^{H}\right|,
$$

where the product is taken over conjugacy classes of subgroups $H \leq G$.

For finite groups $S_{1}$ and $S_{2}$ we regard a $\left(S_{1}, S_{2}\right)$-biset $\Omega$ as a left $\left(S_{1} \times S_{2}\right)$-set by putting $(g, h) x:=h x g^{-1}$ for $g \in S_{1}, h \in S_{2}$ and $x \in \Omega$. This assignment preserves isomorphism classes and we obtain an injection

$$
A\left(S_{1}, S_{2}\right) \longrightarrow A\left(S_{1} \times S_{2}\right)
$$

sending a basis element $[P, \psi]$ to $\left[\left(S_{1} \times S_{2}\right) / \Delta_{P}^{\psi}\right]$. For a subgroup $Q \leq S_{1} \times S_{2}$, this allows us to define $\Omega Q$ as the fixed-point set of $\Omega$ under the action of $Q$. By linear extension and $p$-completion we get a well defined $\mathbb{Z}_{p}^{\wedge}$-module homomorphism

$$
A\left(S_{1}, S_{2}\right)_{p}^{\wedge} \longrightarrow \mathbb{Z}_{p}^{\wedge}, \quad \Omega \mapsto\left|\Omega^{Q}\right|,
$$

depending only on the conjugacy class of $Q$. On basis elements we have

$$
\left|[P, \psi]^{Q}\right|=\left|\left(\left(S_{1} \times S_{2}\right) / \Delta_{P}^{\psi}\right){ }^{Q}\right|=\left|\Delta_{P}^{\psi} \backslash N_{S_{1} \times S_{2}}\left(Q, \Delta_{P}^{\psi}\right)\right|=\frac{\left|N_{S_{1} \times S_{2}}\left(Q, \Delta_{P}^{\psi}\right)\right|}{|P|} .
$$

Lemma 2.6 Let $S_{1}$ and $S_{2}$ be finite $p$-groups. Then the $\mathbb{Z}_{p}^{\wedge}$-module homomorphism

$$
A\left(S_{1}, S_{2}\right)_{p}^{\wedge} \longrightarrow \prod_{[P, \psi]} \mathbb{Z}_{p}^{\wedge}, \quad \Omega \mapsto \prod_{[P, \psi]} \mid \Omega^{\Delta_{P}^{\psi} \mid},
$$

where the product is taken over conjugacy classes of $\left(S_{1}, S_{2}\right)$-pairs, is injective.

Proof Being a composition of two injective homomorphisms, the $\mathbb{Z}$-module homomorphism

$$
A\left(S_{1}, S_{2}\right) \longrightarrow A\left(S_{1} \times S_{2}\right) \longrightarrow \prod_{[Q]} \mathbb{Z}, \quad \Omega \mapsto \prod_{[Q]}\left|\Omega^{Q}\right|,
$$


where the product runs over conjugacy classes of subgroups $Q \leq S_{1} \times S_{2}$, is itself injective. Noting that the collection of graphs of $\left(S_{1}, S_{2}\right)$-pairs is closed under conjugation in $S_{1} \times S_{2}$ and taking subgroups, we see that for a $\left(S_{1}, S_{2}\right)$-pair $(P, \psi)$, we have

$$
N_{S_{1} \times S_{2}}\left(Q, \Delta_{P}^{\psi}\right)=\varnothing
$$

and consequently

$$
\left|[P, \psi]^{Q}\right|=0
$$

if $Q$ is not the graph of an $\left(S_{1}, S_{2}\right)$-pair. We conclude that the restriction to a $\mathbb{Z}$-module homomorphism

$$
A\left(S_{1}, S_{2}\right) \longrightarrow \prod_{[P, \psi]} \mathbb{Z}, \quad \Omega \mapsto \prod_{[P, \psi]}\left|\Omega^{\Delta_{P}^{\psi}}\right|,
$$

is injective, and it remains so after $p$-completion.

\section{Fusion subconjugacy}

In this section we introduce the notion of fusion subconjugacy for subgroups of a finite $p$-group $S$ and for $\left(S_{1}, S_{2}\right)$-pairs. This induces a filtration on the $p$-completed Burnside module $A\left(S_{1}, S_{2}\right)_{p}^{\wedge}$ and consequently of the group $\left\{B S_{1}, B S_{2}\right\}$ of homotopy classes of stable maps. By studying this filtration we will obtain useful information about how homotopy classes of stable maps between classifying spaces of finite $p$-groups behave under composition with stable maps arising from fusion systems over those $p$-groups. The material in this section is presented for $p$-completed Burnside modules because we are mostly interested in that setting. However the analogous results still hold in the uncompleted or $p$-localized case.

Definition 3.1 Let $\mathcal{F}$ be a fusion system over a finite $p$-group $S$, and let $P$ and $Q$ be subgroups of $S$.

- We say that $Q$ is $\mathcal{F}$-subconjugate to $P$, and write $Q \underset{\mathcal{F}}{\precsim} P$, if there exists a morphism $\varphi \in \operatorname{Hom}_{\mathcal{F}}(Q, P)$.

- We say that $Q$ is $\mathcal{F}$-conjugate to $P$, and write $Q \underset{\mathcal{F}}{ } P$ if $Q$ is isomorphic to $P$ in $\mathcal{F}$.

- We say that $Q$ is strictly $\mathcal{F}$-subconjugate to $P$, and write $\underset{\mathcal{F}}{\underset{\gamma}{\prec}} P$ if $Q$ is $\mathcal{F}$-subconjugate to $P$, but not $\mathcal{F}$-conjugate to $P$. 
When there is no danger of confusion, we will write $\precsim, \precsim$, and $\sim$ instead of $\underset{\mathcal{F}}{\precsim} \underset{\mathcal{F}}{\precsim}$ and $\widetilde{\mathcal{F}}$. For the fusion system $\mathcal{F}_{S}$ of $S, \mathcal{F}_{S}$-subconjugacy coincides with $S$-subconjugacy. We make a similar definition for pairs.

Definition 3.2 Let $\mathcal{F}_{1}$ and $\mathcal{F}_{2}$ be fusion systems over finite $p$-groups $S_{1}$ and $S_{2}$, respectively. Let $(P, \psi)$ and $(Q, \rho)$ be two $\left(S_{1}, S_{2}\right)$-pairs.

- We say that $(Q, \rho)$ is $\left(\mathcal{F}_{1}, \mathcal{F}_{2}\right)$-subconjugate to $(P, \psi)$, and write

$$
(Q, \rho) \underset{\left(\mathcal{F}_{1}, \mathcal{F}_{2}\right)}{\precsim}(P, \psi),
$$

if there exist morphisms $\varphi_{1} \in \operatorname{Hom}_{\mathcal{F}_{1}}(Q, P)$ and $\varphi_{2} \in \operatorname{Hom}_{\mathcal{F}_{2}}(\rho(Q), \psi(P))$ such that the following diagram commutes

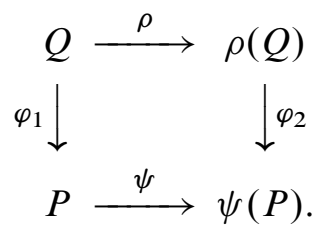

- We say that $(Q, \rho)$ is $\left(\mathcal{F}_{1}, \mathcal{F}_{2}\right)$-conjugate to $(P, \psi)$, and write

$$
(Q, \rho) \underset{\left(\mathcal{F}_{1}, \mathcal{F}_{2}\right)}{\sim}(P, \psi),
$$

if

$$
(Q, \rho) \underset{\left(\mathcal{F}_{1}, \mathcal{F}_{2}\right)}{\precsim}(P, \psi) \quad \text { and } \quad(P, \psi) \underset{\left(\mathcal{F}_{1}, \mathcal{F}_{2}\right)}{\precsim}(Q, \rho) .
$$

- We say that $(Q, \rho)$ is strictly $\left(\mathcal{F}_{1}, \mathcal{F}_{2}\right)$-subconjugate to $(P, \psi)$, and write

$$
(Q, \rho) \underset{\left(\mathcal{F}_{1}, \mathcal{F}_{2}\right)}{\precsim}(P, \psi),
$$

if $(Q, \rho)$ is $\left(\mathcal{F}_{1}, \mathcal{F}_{2}\right)$-subconjugate to $(P, \psi)$, but not $\left(\mathcal{F}_{1}, \mathcal{F}_{2}\right)$-conjugate to $(P, \psi)$.

When there is no danger of confusion, we will write $\precsim$, ঝ, and $\sim$ instead of $\underset{\left(\mathcal{F}_{1}, \mathcal{F}_{2}\right)}{\precsim}, \underset{\left(\mathcal{F}_{1}, \mathcal{F}_{2}\right)}{\precsim}$ and $\underset{\left(\mathcal{F}_{1}, \mathcal{F}_{2}\right)}{\sim}$. As before, $\left(\mathcal{F}_{S_{1}}, \mathcal{F}_{S_{2}}\right)$-conjugacy agrees with the notion of $\left(S_{1}, S_{2}\right)$-conjugacy defined in Section 2.

Remark 3.3 It is easy to check that $\left(\mathcal{F}_{1}, \mathcal{F}_{2}\right)$-subconjugacy is preserved by $\left(S_{1}, S_{2}\right)$ conjugacy. Therefore we will often say that an isomorphism class of pairs $[Q, \rho]$ is $\left(\mathcal{F}_{1}, \mathcal{F}_{2}\right)$-subconjugate to an isomorphism class $[P, \psi]$ and write

$$
[Q, \rho] \underset{\left(\mathcal{F}_{1}, \mathcal{F}_{2}\right)}{\precsim}[P, \psi] \quad(\text { or }[Q, \rho] \precsim[P, \psi])
$$


if the subconjugacy relation

$$
(Q, \rho) \underset{\left(\mathcal{F}_{1}, \mathcal{F}_{2}\right)}{\precsim}(P, \psi)
$$

holds between any (and hence all) representatives of the classes. Furthermore, we will use the same terminology when we regard $[P, \psi]$ and $[Q, \rho]$ as basis elements of $A\left(S_{1}, S_{2}\right)$ or $A\left(S_{1}, S_{2}\right)_{p}^{\wedge}$. The analogous remark applies to $\left(\mathcal{F}_{1}, \mathcal{F}_{2}\right)$-conjugacy and strict $\left(\mathcal{F}_{1}, \mathcal{F}_{2}\right)$-subconjugacy.

Remark 3.4 Subconjugacy among $\left(S_{1}, S_{2}\right)$-pairs can in fact be regarded as a special case of subconjugacy among subgroups of $S_{1} \times S_{2}$. Recall from [7, Section 1], that in the setting of the definition above, the fusion system $\mathcal{F}_{1} \times \mathcal{F}_{2}$ over $S_{1} \times S_{2}$ is defined by setting $\operatorname{Hom}_{\mathcal{F}_{1} \times \mathcal{F}_{2}}(P, Q)$ to be the morphism set

$$
\left\{\left.\left(\varphi_{1}, \varphi_{2}\right)\right|_{P} \in \operatorname{Hom}(P, Q) \mid \varphi_{i} \in \operatorname{Hom}_{\mathcal{F}_{i}}\left(P_{i}, S_{i}\right), P \leq P_{1} \times P_{2}\right\}
$$

for all $P, Q \leq S_{1} \times S_{2}$. By [7, Lemma 1.5], the fusion system $\mathcal{F}_{1} \times \mathcal{F}_{2}$ is saturated if the fusion systems $\mathcal{F}_{1}$ and $\mathcal{F}_{2}$ are both saturated. For $\left(S_{1}, S_{2}\right)$-pairs $(P, \psi)$ and $\left(P^{\prime}, \psi^{\prime}\right)$, one can check that $\left(P^{\prime}, \psi^{\prime}\right)$ is $\left(\mathcal{F}_{1}, \mathcal{F}_{2}\right)$-subconjugate to $(P, \psi)$ if and only $\Delta_{P^{\prime}}^{\psi^{\prime}}$ is $\left(\mathcal{F}_{1} \times \mathcal{F}_{2}\right)$-subconjugate to $\Delta_{P}^{\psi}$.

It is easy to check that $\left(\mathcal{F}_{1}, \mathcal{F}_{2}\right)$-subconjugacy is a transitive relation. Therefore the $\left(\mathcal{F}_{1}, \mathcal{F}_{2}\right)$-conjugacy classes of $\left(S_{1}, S_{2}\right)$-pairs form a poset under $\left(\mathcal{F}_{1}, \mathcal{F}_{2}\right)$-subconjugacy. Since $\left(S_{1}, S_{2}\right)$-conjugacy classes of $\left(S_{1}, S_{2}\right)$-pairs form a $\mathbb{Z}_{p}^{\wedge}$-basis for the $\mathbb{Z}_{p}^{\wedge}$-module $A\left(S_{1}, S_{2}\right)_{p}^{\wedge}$, this leads us to a poset-indexed filtration as defined below.

Definition 3.5 Let $\mathcal{F}_{1}$ and $\mathcal{F}_{2}$ be fusion systems over finite $p$-groups $S_{1}$ and $S_{2}$, respectively. Let $(P, \psi)$ be a $\left(S_{1}, S_{2}\right)$-pair.

- Let $M\left(\precsim[P, \psi], \mathcal{F}_{1}, \mathcal{F}_{2}\right)$ denote the submodule of $A\left(S_{1}, S_{2}\right)_{p}^{\wedge}$ generated by the basis elements $[Q, \rho]$ such that

$$
[Q, \rho] \underset{\left(\mathcal{F}_{1}, \mathcal{F}_{2}\right)}{\precsim}[P, \psi] .
$$

- Let $M\left(\sim[P, \psi], \mathcal{F}_{1}, \mathcal{F}_{2}\right)$ denote the submodule of $A\left(S_{1}, S_{2}\right)_{p}^{\wedge}$ generated by the basis elements $[Q, \rho]$ such that

$$
[Q, \rho] \underset{\left(\mathcal{F}_{1}, \mathcal{F}_{2}\right)}{\sim}[P, \psi] .
$$

- Let $M\left(\precsim[P, \psi], \mathcal{F}_{1}, \mathcal{F}_{2}\right)$ denote the submodule of $A\left(S_{1}, S_{2}\right)_{p}^{\wedge}$ generated by the basis elements $[Q, \rho]$ such that

$$
[Q, \rho] \underset{\left(\mathcal{F}_{1}, \mathcal{F}_{2}\right)}{\precsim}[P, \psi] .
$$


When the fusion systems $\mathcal{F}_{1}$ and $\mathcal{F}_{2}$ are clear from the context, and there is no danger of confusion, we will write $M(\precsim[P, \psi]), M(\sim[P, \psi])$ and $M(\precsim[P, \psi])$ instead of $M\left(\precsim[P, \psi], \mathcal{F}_{1}, \mathcal{F}_{2}\right), M\left(\sim[P, \psi], \mathcal{F}_{1}, \mathcal{F}_{2}\right)$ and $M\left(\precsim[P, \psi], \mathcal{F}_{1}, \mathcal{F}_{2}\right)$.

The stable selfmaps of a finite $p$-group arising from morphisms in a fusion system are of special importance in this paper. We therefore consider the corresponding subring of $A(S, S)_{p}^{\wedge}$.

Definition 3.6 Let $\mathcal{F}$ be a fusion system over a finite $p$-group $S$. We denote by $A_{\mathcal{F}}(S, S)$ the submodule of $A(S, S)$ generated by the basis elements $[P, \varphi]$ where $\varphi \in \operatorname{Hom}_{\mathcal{F}}(P, S)$.

After $p$-completion we obtain a submodule $A_{\mathcal{F}}(S, S)_{p}^{\wedge}$ of $A(S, S)_{p}^{\wedge}$, again generated by the basis elements $[P, \varphi]$ where $\varphi \in \operatorname{Hom}_{\mathcal{F}}(P, S)$.

Remark 3.7 One can check that

$A_{\mathcal{F}}(S, S)_{p}^{\wedge}=M\left(\precsim[S, i d], \mathcal{F}_{S}, \mathcal{F}\right)=M(\precsim[S, i d], \mathcal{F}, \mathcal{F})=M\left(\precsim[S, i d], \mathcal{F}, \mathcal{F}_{S}\right)$.

Under composition, the $\mathbb{Z}_{p}^{\wedge}$-module $A\left(S_{1}, S_{2}\right)_{p}^{\wedge}$ becomes a left $A\left(S_{2}, S_{2}\right)_{p}^{\wedge}$-module and a right $A\left(S_{1}, S_{1}\right)_{p}^{\wedge}$-module. The filtration in Definition 3.5 is useful to us mainly because of the following lemma.

Lemma 3.8 Let $\mathcal{F}_{1}$ and $\mathcal{F}_{2}$ be fusion systems over the finite $p$-groups $S_{1}$ and $S_{2}$, respectively. The following hold for every $\left(S_{1}, S_{2}\right)$-pair $(P, \psi)$ :

(a) $A_{\mathcal{F}_{2}}\left(S_{2}, S_{2}\right)_{p}^{\wedge} \circ M(\precsim[P, \psi]) \subseteq M(\precsim[P, \psi])$,

(b) $A_{\mathcal{F}_{2}}\left(S_{2}, S_{2}\right)_{p}^{\wedge} \circ M(\precsim[P, \psi]) \subseteq M(\precsim[P, \psi])$,

(c) $M(\precsim[P, \psi]) \circ A_{\mathcal{F}_{1}}\left(S_{1}, S_{1}\right)_{p}^{\wedge} \subseteq M(\precsim[P, \psi])$,

(d) $M(\precsim[P, \psi]) \circ A_{\mathcal{F}_{1}}\left(S_{1}, S_{1}\right)_{p}^{\wedge} \subseteq M(\precsim[P, \psi])$.

Proof We prove parts (a) and (b), and leave the proofs of (c) and (d), which are similar, to the reader.

First we show that for any $\left(S_{1}, S_{2}\right)$-pair $(Q, \rho)$ and any basis element $[T, \varphi]$ of $A_{\mathcal{F}_{2}}\left(S_{2}, S_{2}\right)_{p}^{\wedge}$, we have

$$
[T, \varphi] \circ[Q, \rho] \in M(\precsim[Q, \rho]) .
$$

Indeed, by the double coset formula,

$$
[T, \varphi] \circ[Q, \rho]=\sum_{x \in T \backslash S_{2} / \rho(Q)}\left[\rho^{-1}\left(\rho(Q) \cap T^{x}\right), \varphi \circ c_{x} \circ \rho\right],
$$


and it suffices to prove that

$$
\left[\rho^{-1}\left(\rho(Q) \cap T^{x}\right), \varphi \circ c_{x} \circ \rho\right] \precsim[Q, \rho]
$$

for each $x \in S_{2}$. But this is clear by the diagram

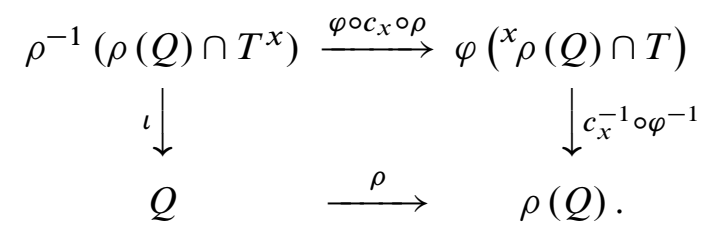

To prove part (a), let $[Q, \rho] \precsim[P, \psi]$. By the preceding observation we get

$$
[T, \varphi] \circ[Q, \rho] \in M(\precsim[Q, \rho]) \subseteq M(\precsim[P, \psi]) .
$$

Letting $[T, \varphi]$ and $[Q, \rho]$ vary over all basis elements of $A_{\mathcal{F}_{2}}\left(S_{2}, S_{2}\right)_{p}^{\wedge}$ and $M(\precsim[P, \psi])$, we get the desired result.

Similarly, part (b) follows upon noting that for a basis element $[Q, \rho]$ of $M(\precsim[P, \psi])$ and a basis element $[T, \varphi]$ of $A_{\mathcal{F}_{2}}\left(S_{2}, S_{2}\right)_{p}^{\wedge}$, we have

$$
[T, \varphi] \circ[Q, \rho] \in M(\precsim[Q, \rho]) \subseteq M(\precsim[P, \psi]) .
$$

We have the following structural corollaries.

Corollary 3.9 Let $\mathcal{F}$ be a fusion system over a finite $p$-group $S$. Then $A_{\mathcal{F}}(S, S)_{p}^{\wedge}$ is a subring of $A(S, S)_{p}^{\wedge}$. Similarly $A_{\mathcal{F}}(S, S)$ is a subring of $A(S, S)$.

Proof This follows from Remark 3.7 and Lemma 3.8. The same proof works for the last statement.

Corollary 3.10 Let $\mathcal{F}_{1}$ and $\mathcal{F}_{2}$ be fusion systems over the finite $p$-groups $S_{1}$ and $S_{2}$, respectively. For every $\left(S_{1}, S_{2}\right)$-pair $(P, \psi)$, the $\mathbb{Z}_{p}^{\wedge}$-modules $M(\precsim[P, \psi])$ and $M(\precsim[P, \psi])$ are left modules over $A_{\mathcal{F}_{2}}\left(S_{2}, S_{2}\right)_{p}^{\wedge}$ and right modules over $A_{\mathcal{F}_{1}}\left(S_{1}, S_{1}\right)_{p}^{\wedge}$.

Definition 3.11 Let $\mathcal{F}$ be a fusion system over a finite $p$-group $S$, and let $\Omega \in A(S, S)_{p}^{\wedge}$. We say that $\Omega$ is right $\mathcal{F}$-stable if for every $P \leq S$ and every $\varphi \in \operatorname{Hom}_{\mathcal{F}}(P, S)$ we have

$$
\Omega \circ[P, \varphi]_{P}^{S}=\Omega \circ\left[P, \iota_{P}\right]_{P}^{S}
$$

Algebraic $8 \mathcal{G}$ Geometric Topology, Volume 6 (2006) 
in $A(P, S)_{p}^{\wedge}$. Similarly we say that $\Omega$ is left $\mathcal{F}$-stable if for every $P \leq S$ and $\varphi \in \operatorname{Hom}_{\mathcal{F}}(P, S)$ we have

$$
\left[\varphi(P), \varphi^{-1}\right]_{S}^{P} \circ \Omega=\left[P, i d_{P}\right]_{S}^{P} \circ \Omega
$$

in $A(S, P)_{p}^{\wedge}$.

When $\Omega$ is represented by a $(S, S)$-biset $X$, the right $\mathcal{F}$-stability condition means that the restriction of $X$ to a $(P, S)$-biset via $\varphi$ is isomorphic to the restriction of $X$ via the inclusion $P \hookrightarrow S$, while left stability means that the restriction of $X$ to a $(S, P)$-biset via $\varphi$ is isomorphic to the restriction of $X$ via the inclusion.

We will later define a similar notion of fusion stability for maps between stable classifying spaces of $p$-groups.

Lemma 3.12 Let $\mathcal{F}_{1}$ and $\mathcal{F}_{2}$ be fusion systems over the finite $p$-groups $S_{1}$ and $S_{2}$, respectively, let $\Omega_{1} \in A\left(S_{1}, S_{1}\right)_{p}^{\wedge}$ be left $\mathcal{F}_{1}-$ stable, and let $\Omega_{2} \in A\left(S_{2}, S_{2}\right)_{p}^{\wedge}$ be right $\mathcal{F}_{2}$-stable. If the $\left(S_{1}, S_{2}\right)$-pairs $(P, \psi)$ and $(Q, \rho)$ are $\left(\mathcal{F}_{1}, \mathcal{F}_{2}\right)$-conjugate, then

$$
\Omega_{2} \circ[Q, \rho] \circ \Omega_{1}=\Omega_{2} \circ[P, \psi] \circ \Omega_{1} .
$$

Proof Let $\tilde{\psi}: P \rightarrow \psi(P)$ denote the restriction of $\psi$ to its image. Since $(P, \psi)$ and $(Q, \rho)$ are conjugate, there exist isomorphisms $\varphi_{1} \in \operatorname{Hom}_{\mathcal{F}_{1}}(P, Q)$ and $\varphi_{2} \in \operatorname{Hom}_{\mathcal{F}_{2}}(\psi(P), \rho(Q))$ such that

$$
\rho \circ \varphi_{1}=\iota_{\rho(Q)} \circ \varphi_{2} \circ \tilde{\psi} .
$$

Using stability, and recalling the simple description of the double coset formula for composable morphisms in (3), we now obtain

$$
\begin{aligned}
\Omega_{2} \circ & {[Q, \rho]_{S_{1}}^{S_{2}} \circ \Omega_{1} } \\
& =\Omega_{2} \circ\left[Q, \iota_{\rho(Q)} \circ \varphi_{2} \circ \tilde{\psi} \circ \varphi_{1}^{-1}\right]_{S_{1}}^{S_{2}} \circ \Omega_{1} \\
& =\Omega_{2} \circ\left(\left[\psi(P), \iota_{\rho}(Q) \circ \varphi_{2}\right]_{\psi(P)}^{S_{2}} \circ[P, \tilde{\psi}]_{P}^{\psi(P)} \circ\left[Q, \varphi_{1}^{-1}\right]_{S_{1}}^{P}\right) \circ \Omega_{1} \\
& =\left(\Omega_{2} \circ\left[\psi(P), \iota_{\rho}(Q) \circ \varphi_{2}\right]_{\psi(P)}^{S_{2}}\right) \circ[P, \tilde{\psi}]_{P}^{\psi(P)} \circ\left(\left[\varphi(P), \varphi_{1}^{-1}\right]_{S_{1}}^{P} \circ \Omega_{1}\right) \\
& =\left(\Omega_{2} \circ\left[\psi(P), \iota_{\psi(P)}\right]_{\psi(P)}^{S_{2}}\right) \circ[P, \tilde{\psi}]_{P}^{\psi(P)} \circ\left(\left[P, i d_{P}\right]_{S_{1}}^{P} \circ \Omega_{1}\right) \\
& \left.=\Omega_{2} \circ\left(\left[\psi(P), \iota_{\psi(P)}\right]_{\psi(P)}^{S_{2}} \circ[P, \tilde{\psi}]_{P}^{\psi(P)} \circ\left[P, i d_{P}\right]_{S_{1}}^{P}\right)\right) \circ \Omega_{1} \\
& =\Omega_{2} \circ[P, \psi]_{S_{1}}^{S_{2}} \circ \Omega_{1} .
\end{aligned}
$$

This completes the proof. 


\section{Characteristic idempotents}

In this section, and for the rest of the paper, we restrict our attention to saturated fusion systems. For a saturated fusion system $\mathcal{F}$ over a finite $p$-group $S$, we will prove the existence of an idempotent $\omega \in A(S, S)_{p}^{\wedge}$, related to $\mathcal{F}$ through properties made precise in Definition 4.3 below. These properties, and their importance, were originally recognized by Linckelmann-Webb for bisets. It is the careful analysis of $\omega$ which will allow us to produce the main results of this paper. In later sections we will see that $\omega$ is uniquely determined by $\mathcal{F}$ and that it characterizes the fusion system $\mathcal{F}$, thus justifying the term characteristic idempotent.

In [7, Section 5], Broto-Levi-Oliver determined the cohomological structure of a $p$-local finite group $(S, \mathcal{F}, \mathcal{L})$. In short, they proved that in cohomology, the natural inclusion $\theta: B S \rightarrow|\mathcal{L}|_{p}^{\wedge}$ induces an isomorphism

$$
H^{*}\left(|\mathcal{L}|_{p}^{\wedge}\right) \stackrel{\cong}{\longrightarrow} H^{*}(\mathcal{F}) \subseteq H^{*}(B S)
$$

where

$$
H^{*}(\mathcal{F}):=\varliminf_{\mathcal{F}} H^{*}(B(-))
$$

is the "ring of stable elements for $\mathcal{F}$ ", regarded as a subring of $H^{*}(B S)$, via the identification

$$
H^{*}(\mathcal{F}) \cong\left\{x \in H^{*}(B S) \mid B \varphi^{*}(x)=B \iota_{P}^{*}(x) \text { for all } P \leq S, \varphi \in \operatorname{Hom}_{\mathcal{F}}(P, S)\right\} .
$$

One of the key ingredients in their proof is the construction of a characteristic biset $\Omega \in A^{+}(S, S)$, as defined below. We take advantage of their construction and produce our characteristic idempotent by showing the convergence of a judiciously chosen subsequence of the sequence

$$
[\Omega],[\Omega]^{2},[\Omega]^{3}, \ldots
$$

Definition 4.1 Let $\mathcal{F}$ be a fusion system over a finite $p$-group $S$. We say that an element $\Omega \in A(S, S)$ is a virtual characteristic biset for $\mathcal{F}$ if it has the following properties:

(a') $\Omega \in A_{\mathcal{F}}(S, S)$.

(b1') $\Omega$ is right $\mathcal{F}$-stable.

(b2') $\Omega$ is left $\mathcal{F}$-stable.

(c') $\epsilon(\Omega) \equiv 1(\bmod p)$.

If in addition $\Omega \in A^{+}(S, S)$ then we say that $\Omega$ is a characteristic biset for $\mathcal{F}$. 
We refer to these properties as the Linckelmann-Webb properties as they were first suggested in unpublished work of Linckelmann-Webb [13], although Property (b2') did not feature there. We refer to Properties $\left(\mathrm{b}^{\prime}\right)$ and $\left(\mathrm{b} 2^{\prime}\right)$ collectively as Property $\left(\mathrm{b}^{\prime}\right)$. The Linckelmann-Webb properties mimic the properties of a finite group $G$ with Sylow subgroup $S$ regarded as an $(S, S)$-biset, although some scaling may be required to obtain Property $\left(c^{\prime}\right)$. The importance of the Linckelmann-Webb properties is apparent in the following result.

Proposition $4.2[13 ; 7]$ Let $\mathcal{F}$ be a fusion system over a finite $p$-group $S$. If $\Omega$ is a virtual characteristic biset for $\mathcal{F}$, then the induced map $\alpha(\Omega)^{*}$ in cohomology is an idempotent in End $\left(H^{*}(B S)\right)$, is $H^{*}(\mathcal{F})$-linear and a homomorphism of modules over the Steenrod algebra; and

$$
\operatorname{Im}\left[H^{*}(B S) \stackrel{\alpha(\Omega)^{*}}{\longrightarrow} H^{*}(B S)\right]=H^{*}(\mathcal{F}) .
$$

Proof See the proof of [7, Proposition 5.5].

A characteristic idempotent for a fusion system $\mathcal{F}$ over $S$ is an idempotent in $A(S, S)_{p}^{\wedge}$ with $p$-completed, idempotent analogues of the Linckelmann-Webb properties. This is stated precisely below.

Definition 4.3 Let $\mathcal{F}$ be a fusion system over a finite $p$-group $S$. A characteristic idempotent for $\mathcal{F}$ is an idempotent $\omega \in A(S, S)_{p}^{\wedge}$ with the following properties:

(a) $\omega \in A_{\mathcal{F}}(S, S)_{p}^{\wedge}$.

(b1) $\omega$ is right $\mathcal{F}$-stable.

(b2) $\omega$ is left $\mathcal{F}$-stable.

(c) $\epsilon(\omega)=1$.

We again refer to Properties (b1) and (b2) collectively as Property (b).

The existence of characteristic bisets for saturated fusion systems was established by Broto-Levi-Oliver in [7] through a constructive argument. Although they, like Linckelmann-Webb, did not include Property (b2') in their statement of the result, it is implicit in their construction.

Proposition 4.4 [7, Proposition 5.5] Every saturated fusion system $\mathcal{F}$ over a $p-$ group $S$ has a characteristic $(S, S)$-biset. 
The preceding proposition is the only point in this paper where we rely on the saturation of fusion systems. If we were instead to assume that every fusion system in sight has a characteristic biset, then the construction of characteristic idempotents and classifying spectra, as well as the proof of their properties still go through. It is an interesting question whether this really amounts to weakening our hypothesis. That is, whether the existence of a characteristic biset for a fusion system $\mathcal{F}$ implies that $\mathcal{F}$ is saturated.

We now proceed by a sequence of lemmas about $(S, S)$-bisets to produce the characteristic idempotent.

Lemma 4.5 Let $\Omega$ and $\Lambda$ be two (virtual) characteristic bisets for a fusion system $\mathcal{F}$ over a finite $p$-group $S$. Then $\Omega \circ \Lambda$ is also a (virtual) characteristic biset for $\mathcal{F}$. In particular, any power of $\Omega$ is a (virtual) characteristic biset for $\mathcal{F}$.

Proof That $\Omega \circ \Lambda$ has Property ( $\left.\mathrm{a}^{\prime}\right)$ follows from Corollary 3.9. To see that $\Omega \circ \Lambda$ has Property $\left(\mathrm{b}^{\prime}\right)$, we note that for $P \leq S$ and $\varphi \in \operatorname{Hom}_{\mathcal{F}}(P, S)$ we have

$$
(\Omega \circ \Lambda) \circ[P, \varphi]_{P}^{S}=\Omega \circ\left(\Lambda \circ[P, \varphi]_{P}^{S}\right)=\Omega \circ\left(\Lambda \circ\left[P, \iota_{P}\right]_{P}^{S}\right)=(\Omega \circ \Lambda) \circ\left[P, \iota_{P}\right]_{P}^{S},
$$

and similarly

$$
\left[\varphi(P), \varphi^{-1}\right]_{S}^{P} \circ(\Omega \circ \Lambda)=\left[P, i d_{P}\right]_{S}^{P} \circ(\Omega \circ \Lambda) .
$$

Property $\left(\mathrm{c}^{\prime}\right)$ is clearly preserved since $\epsilon$ is multiplicative. The final statement now follows by induction.

Lemma 4.6 Let $\Omega \in A(S, S)$. Then there exists an $M>0$ such that $\Omega^{M}$ is idempotent $\bmod p$.

Proof Let $\bar{\Omega}$ denote the image of $\Omega$ under the projection

$$
A(S, S) \rightarrow A(S, S) / p A(S, S) .
$$

It is equivalent to show that $\bar{\Omega}^{M}$ is idempotent for some $M>0$. Now, $A(S, S)$ is a finitely generated $\mathbb{Z}$-module and hence $A(S, S) / p A(S, S)$ is finite. Consider the sequence

$$
\bar{\Omega}, \bar{\Omega}^{2}, \bar{\Omega}^{3}, \ldots
$$

in $A(S, S) / p A(S, S)$. By the pigeonhole principle there must be numbers $N, t>0$ such that $\bar{\Omega}^{N}=\bar{\Omega}^{N+t}$. It follows that

$$
\bar{\Omega}^{n}=\bar{\Omega}^{n+t}
$$

for all $n \geq N$. Now take $m \geq 0$ such that $m t>N$ and put $M:=m t$. Then

$$
\bar{\Omega}^{2 M}=\bar{\Omega}^{M+m t}=\bar{\Omega}^{M+(m-1) t}=\cdots=\bar{\Omega}^{M+t}=\bar{\Omega}^{M} .
$$


The following two lemmas were suggested to the author by Bob Oliver. Although they hold for any $p$-torsion-free ring, we will state them only for $A(S, S)$.

Lemma 4.7 If $\Omega \in A(S, S)$ is idempotent $\bmod p^{k}$, where $k>0$, then $\Omega^{p}$ is idempotent $\bmod p^{k+1}$.

Proof Put $q:=p^{k}$. By assumption we can write

$$
\Omega^{2}=\Omega+q \Lambda
$$

for some $\Lambda \in A(S, S)$. It follows that

$$
\Omega^{2}+q \Omega \Lambda=\Omega(\Omega+q \Lambda)=\Omega^{3}=(\Omega+q \Lambda) \Omega=\Omega^{2}+q \Lambda \Omega,
$$

so

$$
q \Omega \Lambda=q \Lambda \Omega .
$$

Since $A(S, S)$ is torsion-free as a $\mathbb{Z}$-module, we deduce that $\Omega$ and $\Lambda$ commute. This allows us to apply the binomial formula to (4) and get

$$
\Omega^{2 p}=\Omega^{p}+\left(\begin{array}{c}
p \\
1
\end{array}\right) \Omega^{p-1} q \Lambda+\left(\begin{array}{l}
p \\
2
\end{array}\right) \Omega^{p-2} q^{2} \Lambda^{2}+\cdots+\left(\begin{array}{c}
p \\
p-1
\end{array}\right) \Omega q^{p-1} \Lambda^{p-1}+q^{p} \Lambda^{p} .
$$

A brief inspection of the coefficients occurring on the right hand side, taking into account that $p$ divides $q$ since $k>0$, shows that we can therefore write

$$
\Omega^{2 p}=\Omega^{p}+p q \Lambda^{\prime}
$$

for some $\Lambda^{\prime} \in A(S, S)$. Since $p q=p^{k+1}$ we deduce that $\Omega^{p}$ is idempotent $\bmod p^{k+1}$.

Lemma 4.8 If $\Omega \in A(S, S)$ is idempotent $\bmod p$, then the sequence

$$
\Omega, \Omega^{p}, \Omega^{p^{2}}, \ldots
$$

converges in $A(S, S)_{p}^{\wedge}$. Furthermore the limit is idempotent.

Proof By Lemma 4.7 and induction, $\Omega^{p^{k}}$ is idempotent $\bmod p^{k+1}$ for each $k \geq 0$. That is to say,

$$
\Omega^{2 p^{k}}-\Omega^{p^{k}} \in p^{k+1} A(S, S)
$$

for $k \geq 0$. By induction it follows that

$$
\Omega^{n p^{k}}-\Omega^{p^{k}} \in p^{k+1} A(S, S)
$$

Algebraic 83 Geometric Topology, Volume 6 (2006) 
for $k \geq 0, n>0$. In particular

$$
\Omega^{p^{l}}-\Omega^{p^{k}} \in p^{k+1} A(S, S)
$$

when $l \geq k>0$, so

$$
\Omega, \Omega^{p}, \Omega^{p^{2}}, \ldots
$$

is a Cauchy sequence in the $p$-adic topology of $A(S, S)$. Hence it converges to a unique element $\omega \in A(S, S)_{p}^{\wedge}$. Since the multiplication in $A(S, S)$ is continuous with respect to the $p$-adic topology, $\omega^{2}$ is the limit of the sequence

$$
\Omega^{2}, \Omega^{2 p}, \Omega^{2 p^{2}}, \ldots
$$

Idempotence of $\omega$ now follows by taking the limit of (5) over $k$.

We can now prove the main result of this section.

Proposition 4.9 Every saturated fusion system has a characteristic idempotent.

Proof Let $\mathcal{F}$ be a saturated fusion system over a finite $p$-group $S$. Take a characteristic $(S, S)$-biset $\Omega$ as given by Proposition 4.4. By Lemmas 4.6 and 4.5 we may assume that $\Omega$ is idempotent $\bmod p$. By Lemma 4.8 the sequence

$$
\Omega, \Omega^{p}, \Omega^{p^{2}}, \ldots
$$

converges to an idempotent $\omega \in A(S, S)_{p}^{\wedge}$. We show that $\omega$ has the LinckelmannWebb properties.

By an induction similar to that in Lemma 4.7 one can show that $\epsilon(\Omega) \equiv 1(\bmod p)$ implies that $\epsilon\left(\Omega^{p^{k}}\right) \equiv 1\left(\bmod p^{k+1}\right)$ for $k \geq 0$. It follows that $\epsilon(\omega)=1$, proving (c).

It is not hard to see that $A_{\mathcal{F}}(S, S)$ is a closed subspace of $A(S, S)$ in the $p$-adic topology and hence that $A_{\mathcal{F}}(S, S)_{p}^{\wedge}$ is a closed subspace of $A(S, S)_{p}^{\wedge}$. Since each $\Omega^{n}$ is in $A_{\mathcal{F}}(S, S)$ by Corollary 3.9, it follows that the limit $\omega$ is in $A_{\mathcal{F}}(S, S)_{p}^{\wedge}$, proving (a).

Let $P \leq S$ and $\varphi \in \operatorname{Hom}_{\mathcal{F}}(P, S)$. By Property (b1') we have

$$
\Omega \circ[P, \varphi]_{P}^{S}=\Omega \circ\left[P, \iota_{P}\right]_{P}^{S}
$$

and consequently

$$
\Omega^{p^{k}} \circ[P, \varphi]_{P}^{S}=\Omega^{p^{k}} \circ\left[P, \iota_{P}\right]_{P}^{S},
$$

Algebraic 83 Geometric Topology, Volume 6 (2006) 
for all $k \geq 0$. Since the pairing

$$
\circ: A(S, S) \times A(P, S) \rightarrow A(P, S)
$$

is continuous in the $p$-adic topology on the relevant $\mathbb{Z}$-modules, we can take limits to get

$$
\omega \circ[P, \varphi]_{P}^{S}=\omega \circ\left[P, \iota_{P}\right]_{P}^{S},
$$

proving (b1). Property (b2) follows similarly from Property (b2').

\section{Properties of characteristic idempotents}

In this section we perform a further study of the characteristic idempotents introduced in the previous section. We discover that the effect of multiplicaton by a characteristic idempotent on $A\left(S_{1}, S_{2}\right)_{p}^{\wedge}$ essentially amounts to quotienting out fusion conjugacy in the source or target, as appropriate. This allows us to glean important information about the structure of a characteristic idempotent in the $[P, \varphi]$-basis, which allows us to prove its uniqueness, and will also prove surprisingly useful for proving later naturality results.

Proposition 5.1 Let $\mathcal{F}_{1}$ and $\mathcal{F}_{2}$ be saturated fusion systems over the finite $p$-groups $S_{1}$ and $S_{2}$, respectively, and let $\omega_{1}$ and $\omega_{2}$ be characteristic idempotents of $\mathcal{F}_{1}$ and $\mathcal{F}_{2}$, respectively. If the $\left(S_{1}, S_{2}\right)$-pairs $(P, \psi)$ and $(Q, \rho)$ are $\left(\mathcal{F}_{1}, \mathcal{F}_{2}\right)$-conjugate, then

$$
\omega_{2} \circ[Q, \rho] \circ \omega_{1}=\omega_{2} \circ[P, \psi] \circ \omega_{1} .
$$

Proof Since $\omega_{1}$ is left $\mathcal{F}_{1}$-stable and $\omega_{2}$ is right $\mathcal{F}_{2}$-stable, this is a special case of Lemma 3.12.

Although the following proposition may be of limited interest in its own right, it is the central result of this paper.

Proposition 5.2 Let $\mathcal{F}_{1}$ and $\mathcal{F}_{2}$ be saturated fusion systems over the finite $p$-groups $S_{1}$ and $S_{2}$, respectively and let $\omega_{1}$ and $\omega_{2}$ be characteristic idempotents of $\mathcal{F}_{1}$ and $\mathcal{F}_{2}$, respectively. Let $I$ be the set of $\left(\mathcal{F}_{1}, \mathcal{F}_{2}\right)$-conjugacy classes of $\left(S_{1}, S_{2}\right)$-pairs, and pick a representative $\left(P_{i}, \psi_{i}\right)$ for each $i \in I$. Then the collection

$$
\left\{\omega_{2} \circ\left[P_{i}, \psi_{i}\right] \circ \omega_{1} \mid i \in I\right\}
$$

forms a $\mathbb{Z}_{p}^{\wedge}$-basis for $\omega_{2} \circ A\left(S_{1}, S_{2}\right)_{p}^{\wedge} \circ \omega_{1}$. 
Proof It follows from Proposition 5.1 that $\omega_{2} \circ A\left(S_{1}, S_{2}\right)_{p}^{\wedge} \circ \omega_{1}$ is spanned by the collection, so it suffices to prove linear independence. By Property (b) of characteristic idempotents and Lemma 3.8 we have

$$
\omega_{2} \circ\left[P_{i}, \psi_{i}\right] \circ \omega_{1} \in M\left(\precsim\left[P_{i}, \psi_{i}\right]\right)
$$

for each $i \in I$. Note however, that

$$
\epsilon\left(\omega_{2} \circ\left[P_{i}, \psi_{i}\right] \circ \omega_{1}\right)=\epsilon\left(\omega_{2}\right) \cdot \epsilon\left(\left[P_{i}, \psi_{i}\right]\right) \cdot \epsilon\left(\omega_{1}\right)=1 \cdot\left|S_{1} / P_{i}\right| \cdot 1=\left|S_{1} / P_{i}\right|,
$$

whereas

$$
\epsilon\left(M\left(\precsim\left[P_{i}, \psi_{i}\right]\right)\right) \subseteq p\left|S_{1} / P_{i}\right| \mathbb{Z}_{p}^{\wedge}
$$

Therefore,

$$
\omega_{2} \circ\left[P_{i}, \psi_{i}\right] \circ \omega_{1} \in M\left(\precsim\left[P_{i}, \psi_{i}\right]\right) \backslash M\left(\precsim\left[P_{i}, \psi_{i}\right]\right)
$$

for each $i \in I$.

Now, let $c_{i} \in \mathbb{Z}_{p}^{\wedge}$ for each $i \in I$ and assume that

$$
\sum_{i \in I} c_{i} \cdot\left(\omega_{2} \circ\left[P_{i}, \psi_{i}\right] \circ \omega_{1}\right)=0 .
$$

Put

$$
I^{\prime}=\left\{i \in I \mid c_{i} \neq 0\right\}
$$

If $I^{\prime}$ is nonempty, then let $j$ be a maximal element of $I^{\prime}$ regarded as a poset under $\left(\mathcal{F}_{1}, \mathcal{F}_{2}\right)$-subconjugacy. By (7) there is a $\left(S_{1}, S_{2}\right)$-pair $(Q, \rho) \sim\left(P_{j}, \psi_{j}\right)$ such that

$$
\chi_{[Q, \rho]}\left(\omega_{2} \circ\left[P_{j}, \psi_{j}\right] \circ \omega_{1}\right) \neq 0 .
$$

On the other hand, for $i \in I^{\prime} \backslash\{j\}$ the maximality of $j$ implies that $[Q, \rho]$ is not $\left(\mathcal{F}_{1}, \mathcal{F}_{2}\right)$-subconjugate to $\left(P_{i}, \psi_{i}\right)$. Hence

$$
\chi_{[Q, \rho]}\left(M\left(\precsim\left[P_{i}, \psi_{i}\right]\right)\right)=0
$$

and in particular

$$
\chi_{[Q, \rho]}\left(\omega_{2} \circ\left[P_{i}, \psi_{i}\right] \circ \omega_{1}\right)=0
$$

Now we get

$$
\begin{aligned}
\chi_{[Q, \rho]}\left(\sum_{i \in I} c_{i} \cdot\left(\omega_{2} \circ\left[P_{i}, \psi_{i}\right] \circ \omega_{1}\right)\right) & =\sum_{i \in I} c_{i} \cdot \chi_{[Q, \rho]}\left(\omega_{2} \circ\left[P_{i}, \psi_{i}\right] \circ \omega_{1}\right) \\
& =\sum_{i \in I \backslash I^{\prime}} \underbrace{c_{i}}_{=0} \cdot \chi_{[Q, \rho]}\left(\omega_{2} \circ\left[P_{i}, \psi_{i}\right] \circ \omega_{1}\right)
\end{aligned}
$$

Algebraic $8 \mathcal{G}$ Geometric Topology, Volume 6 (2006) 


$$
\begin{aligned}
& +\sum_{i \in I^{\prime} \backslash\{j\}} c_{i} \cdot \underbrace{\chi[Q, \rho]}_{=0}\left(\omega_{2} \circ\left[P_{i}, \psi_{i}\right] \circ \omega_{1}\right) \\
& +\underbrace{c_{j}}_{\neq 0} \cdot \underbrace{\chi[Q, \rho]}_{\neq 0}\left(\omega_{2} \circ\left[P_{j}, \psi_{j}\right] \circ \omega_{1}\right) \\
& \neq 0,
\end{aligned}
$$

contradicting (8). Therefore $I^{\prime}$ must be empty and we conclude that the collection is linearly independent.

Remark 5.3 Since the multiplicative identity $\left[S_{1}, i d\right]$ of $A\left(S_{1}, S_{1}\right)_{p}^{\wedge}$ is a characteristic idempotent for $\mathcal{F}_{S_{1}}$ and $\left[S_{2}, i d\right]$ is a characteristic idempotent for $\mathcal{F}_{S_{2}}$, Propositions 5.1 and 5.2 can also be used to obtain a basis for $\omega_{2} \circ A\left(S_{1}, S_{2}\right)_{p}^{\wedge}$ and $A\left(S_{1}, S_{2}\right)_{p}^{\wedge} \circ \omega_{1}$.

We determine the structure of a characteristic idempotent $\omega$ by carefully analyzing the idempotence relation $\omega \circ \omega=\omega$. In light of the previous proposition, a convenient tool for doing this is the projection

$$
A_{\mathcal{F}}(S, S)_{p}^{\wedge} \longrightarrow \omega A_{\mathcal{F}}(S, S)_{p}^{\wedge}
$$

given by left multiplication by $\omega$. This projection can be easily described by

$$
\Omega=\sum_{[P, \varphi] \lesssim[S, i d]} \chi_{[P, \varphi]}(\Omega) \cdot[P, \varphi] \longmapsto \omega \circ \Omega=\sum_{[P] \lesssim[S]} \chi_{[P]}^{\mathcal{F}}(\Omega) \cdot\left(\omega \circ\left[P, \iota_{P}\right]\right),
$$

where the homomorphisms $\chi_{[P]}^{\mathcal{F}}$ are as in the following definition.

Definition 5.4 Let $\mathcal{F}$ be a fusion system over a finite $p$-group $S$. For each $S$ conjugacy class $[P]$ of subgroups of $S$, let $\chi_{[P]}^{\mathcal{F}}$ and $\chi_{\mathcal{F}}^{[P]}$ be the homomorphisms $A(S, S)_{p}^{\wedge} \rightarrow \mathbb{Z}_{p}^{\wedge}$ given by

$$
\chi_{[P]}^{\mathcal{F}}=\sum_{[P, \varphi]} \underset{\underset{\left(\mathcal{F}_{S}, \mathcal{F}\right)}{\sim}\left[P, \iota_{P}\right]}{ } \chi_{[P, \varphi]}
$$

and

$$
\chi_{\mathcal{F}}^{[P]}=\sum_{[Q, \varphi]} \underset{\left(\mathcal{F}, \tilde{F}_{S}\right)}{ } \chi_{\left[Q, \iota_{P}\right]}[.
$$

Note that similarly we have

$$
\Omega \circ \omega=\sum_{[P] \preccurlyeq[S]} \chi_{\mathcal{F}}^{[P]}(\Omega) \cdot\left(\left[P, \iota_{P}\right] \circ \omega\right) .
$$

Algebraic $\mathcal{B}$ Geometric Topology, Volume 6 (2006) 
The following lemma now effectively allows us to determine the structure of characteristic idempotents.

Lemma 5.5 Let $\mathcal{F}$ be a saturated fusion system over a finite $p$-group $S$ and let $\omega$ be a characteristic idempotent of $\mathcal{F}$. Then

$$
\omega=\omega^{\sim}+\omega^{\precsim},
$$

where

$$
\omega^{\sim}=\frac{1}{\left|\operatorname{Out}_{\mathcal{F}}(S)\right|} \sum_{\varphi \in \operatorname{Out}_{\mathcal{F}}(S)}[S, \varphi] \in M(\sim[S, i d], \mathcal{F}, \mathcal{F})
$$

and

$$
\omega^{\precsim} \in M(\precsim[S, i d], \mathcal{F}, \mathcal{F}) .
$$

Furthermore,

$$
\chi_{[P]}^{\mathcal{F}}(\omega)=0
$$

and

$$
\chi_{\mathcal{F}}^{[P]}(\omega)=0
$$

for all proper subgroups $P<S$.

Proof There is a direct sum of modules

$$
A_{\mathcal{F}}(S, S)_{p}^{\wedge}=M(\precsim[S, i d])=M(\sim[S, i d]) \oplus M(\precsim[S, i d]),
$$

where $M(\underset{\precsim}{\prec}[S, i d])$ is a two-sided ideal of $A_{\mathcal{F}}(S, S)_{p}^{\wedge}$ (by Lemma 3.8), and $M(\sim[S, i d])$ is a subring of $A_{\mathcal{F}}(S, S)_{p}^{\wedge}$ (as can be easily checked). We can therefore uniquely write

$$
\omega=\omega^{\sim}+\omega^{\curvearrowright},
$$

where

$$
\omega^{\sim} \in M(\sim[S, i d])
$$

and

$$
\omega^{\precsim} \in M(\precsim[S, i d]) .
$$

From Properties (a) and (b) of characteristic idempotents, one deduces by standard techniques that $\omega^{\sim}$ must be of the form

$$
\omega^{\sim}=\alpha \sum_{\varphi \in \operatorname{Out}_{\mathcal{F}}(S)}[S, \varphi]
$$

where $\alpha \in \mathbb{Z}_{p}^{\wedge}$. Now,

$$
\omega^{\sim}+\omega^{\precsim}=\omega=\omega^{2}=\left(\omega^{\sim}\right)^{2}+\left(\omega^{\sim} \circ \omega^{\nwarrow}+\omega^{\nwarrow} \circ \omega^{\sim}+\left(\omega^{\precsim}\right)^{2}\right),
$$

Algebraic 83 Geometric Topology, Volume 6 (2006) 
where $\left(\omega^{\sim}\right)^{2} \in M(\sim[S, i d])$ and the second term is in the ideal $M(\precsim[S, i d])$. By uniqueness of such decompositions $\omega^{\sim}$ is therefore an idempotent, and since

$$
\left(\omega^{\sim}\right)^{2}=\alpha \cdot\left|\operatorname{Out}_{\mathcal{F}}(S)\right| \cdot \omega^{\sim},
$$

this implies that

$$
\alpha \in\left\{0,\left|\operatorname{Out}_{\mathcal{F}}(S)\right|^{-1}\right\} .
$$

(Note that $\left|\operatorname{Out}_{\mathcal{F}}(S)\right|$ is prime to $p$ since $\mathcal{F}$ is saturated.) By Property (c) of characteristic idempotents we have

$$
1=\epsilon(\omega)=\epsilon\left(\omega^{\sim}\right)+\epsilon\left(\omega^{\precsim}\right) .
$$

As a special case of (6) we have $\epsilon(M(\precsim[S, i d])) \subseteq p \mathbb{Z}_{p}^{\wedge}$, and it follows that

$$
\alpha \cdot\left|\operatorname{Out}_{\mathcal{F}}(S)\right|=\epsilon\left(\omega^{\sim}\right) \equiv \epsilon(\omega) \equiv 1(\bmod p) .
$$

Hence $\alpha \neq 0$, leaving only the possibility

$$
\omega^{\sim}=\frac{1}{\left|\operatorname{Out}_{\mathcal{F}}(S)\right|} \sum_{\varphi \in \operatorname{Out}_{\mathcal{F}}(S)}[S, \varphi] .
$$

To prove the second claim we start by deducing from Property (b) and the description of $\omega^{\sim}$ above, that

$$
\omega \circ \omega^{\sim}=\omega .
$$

Hence the idempotence of $\omega$ yields

$$
\omega=\omega \circ \omega=\omega \circ\left(\omega^{\sim}+\omega^{\nwarrow}\right)=\omega+\omega \circ \omega^{\nwarrow},
$$

and we get

$$
\omega \circ \omega^{\precsim}=0 .
$$

By Property (a) and Remark 3.7, we can write

$$
\omega^{\precsim}=\sum_{[P, \varphi] \ngtr[S, i d]} \chi_{[P, \varphi]}(\omega) \cdot[P, \varphi],
$$

so

$$
0=\omega \circ \omega^{\precsim}=\sum_{[P]_{\nsim}[S]} \chi_{[P]}^{\mathcal{F}}(\omega) \cdot\left(\omega \circ\left[P, \iota_{P}\right]\right),
$$

and the result for $\chi_{[P]}^{\mathcal{F}}(\omega)$ follows upon noting that the collection

$$
\left\{\omega \circ\left[P, \iota_{P}\right] \mid[P] \precsim[S]\right\}
$$

is linearly independent over $\mathbb{Z}_{p}^{\wedge}$ by Proposition 5.2 and Remark 5.3.

Algebraic 83 Geometric Topology, Volume 6 (2006) 
The result for $\chi_{\mathcal{F}}^{[P]}(\omega)$ is proved similarly by performing the analogous simplifications of $\omega^{\precsim} \circ \omega=0$. We omit the details.

The lemma has an interesting consequence.

Proposition 5.6 Every saturated fusion system has a unique characteristic idempotent.

Proof Let $\mathcal{F}$ be a saturated fusion system over a finite $p$-group $S$. By Proposition $4.9, \mathcal{F}$ has a characteristic idempotent $\omega$. We proceed to prove uniqueness. Recalling that we can write

$$
\omega=\sum_{[P, \varphi] \lesssim[S, i d]} \chi_{[P, \varphi]}(\omega) \cdot[P, \varphi],
$$

the method of proof is to set up a system of linear equations for the coefficients $\chi_{[P, \varphi]}(\omega)$, and show that this system is fully determined and thus uniquely determines the coefficients. Note that we need not show that the system is solvable since the existence of a characteristic idempotent has already been established.

To present the argument it is helpful to index the basis of $A_{\mathcal{F}}(S, S)_{p}^{\wedge}$. Take representatives $P_{0}, P_{1}, \ldots, P_{n}$ for the $S$-conjugacy classes of subgroups of $S$, labelled in such a way that

$$
\left|P_{0}\right| \geq\left|P_{1}\right| \geq \ldots \geq\left|P_{n}\right| \text {. }
$$

For each $i \in\{0,1, \ldots, n\}$, pick representatives $\varphi_{i 0}, \varphi_{i 1}, \ldots, \varphi_{i m_{i}}$ for the $S$-conjugacy classes of homomorphisms in $\operatorname{Hom}_{\mathcal{F}}(P, S)$, now labelled so that $\varphi_{i 0}=\iota_{P_{i}}$. The collection $\left\{\left[P_{i}, \varphi_{i j}\right] \mid 0 \leq i \leq n, 0 \leq j \leq m_{i}\right\}$ is then a basis for $A_{\mathcal{F}}(S, S)_{p}^{\wedge}$. We order the basis through the lexicographic order of the indexing set:

$$
(0,0) \leq(0,1) \leq \cdots \leq\left(0, m_{0}\right) \leq(1,0) \leq(1,1) \leq \cdots \leq\left(n, m_{n}\right) .
$$

This ordering has the property that $\left[P_{i}, \varphi_{i j}\right] \underset{(S, S)}{\precsim}\left[P_{k}, \varphi_{k l}\right]$ implies $(k, l) \leq(i, j)$.

For the remainder of this proof, a pair $(i, j)$ is understood to satisfy $0 \leq i \leq n$ and $1 \leq j \leq m_{i}$. For such a pair $(i, j)$, write

$$
\Delta_{i}^{j}:=\Delta_{P_{i}}^{\varphi_{i j}}
$$

and

$$
c_{i j}:=\chi_{\left[P_{i}, \varphi_{i}^{j}\right]}(\omega)
$$

for short. We will also use the shorthand notation $\sum_{i, j}$ for the double sum $\sum_{i=0}^{n} \sum_{j=0}^{m_{i}}$. Using this notation we can write

$$
\omega=\sum_{i, j} c_{i j}\left[P_{i}, \varphi_{i j}\right]
$$

Algebraic $8 \mathcal{G}$ Geometric Topology, Volume 6 (2006) 
Note that by Lemma 2.6, Property (b1) implies that

$$
\left|\omega^{\Delta_{i}^{j}}\right|=\left|\omega^{\Delta_{i}^{0}}\right|
$$

for all pairs $(i, j)$. This can be rewritten as the equation

$$
\sum_{k, l} c_{k l} \cdot\left(\left|\left[P_{k}, \varphi_{k l}\right]^{\Delta_{i}^{j}}\right|-\left|\left[P_{k}, \varphi_{k l}\right]^{\Delta_{i}^{0}}\right|\right)=0,
$$

which we refer to as Equation $(i, j)$ when $j \neq 0$. When $j=0$, this equation becomes trivial. Instead we consider the equations given by Lemma 5.5. That is, we let Equation $(0,0)$ be the equation

$$
\sum_{j=0}^{m_{0}} c_{0 j}=1
$$

and for $i=1, \ldots, n$, we let Equation $(i, 0)$ be the equation

$$
\sum_{j=0}^{m_{i}} c_{i j}=0
$$

If we now write Equations $(0,0)$ to $\left(i, m_{i}\right)$ one below the other going by the lexicographic order, we obtain a system of equations, which can be represented on matrix form as

$$
A c=b,
$$

where $c$ is a vector with entries $c_{i j}, b$ is a vector with 1 in its first entry and 0 everywhere else, while $A$ is a square matrix whose rows and columns are both indexed by pairs $(i, j)$. The proof is completed by showing that $A$ has nonzero determinant.

There is an obvious way to regard $A$ as a $(n \times n)$ block matrix where the blocks are indexed by $i$. We show that $A$ is in fact a lower triangular block matrix. Since

$$
\left|[Q, \psi]^{\Delta_{R}^{\rho}}\right|=\left|\Delta_{Q}^{\psi} \backslash N_{S \times S}\left(\Delta_{R}^{\rho}, \Delta_{Q}^{\psi}\right)\right|=\frac{\left|N_{S \times S}\left(\Delta_{R}^{\rho}, \Delta_{Q}^{\psi}\right)\right|}{|Q|}
$$

for $(S, S)$-pairs $(Q, \psi)$ and $(R, \rho)$, we see by Remark 3.4 that

$$
\left|[Q, \psi]^{\Delta_{R}^{\rho}}\right| \neq 0
$$

if and only if

$$
[R, \rho] \underset{(\widetilde{S, S)}}{\precsim}[Q, \psi] .
$$

The chosen order of the basis of $A_{\mathcal{F}}(S, S)_{p}^{\wedge}$ therefore guarantees that $A$ is a lower triangular block matrix. 
It now suffices to show that the matrices occurring on the diagonal of $A$ have nonzero determinant. The $i$-th matrix on the diagonal has the form

$$
A_{i}=\left(\begin{array}{ccccccc}
1 & 1 & 1 & & 1 & 1 & 1 \\
-a_{i 0} & a_{i 1} & 0 & \ldots & 0 & 0 & 0 \\
-a_{i 0} & 0 & a_{i 2} & & 0 & 0 & 0 \\
& \vdots & & \ddots & & \vdots & \\
-a_{i 0} & 0 & 0 & & a_{i m_{i}-2} & 0 & 0 \\
-a_{i 0} & 0 & 0 & \ldots & 0 & a_{i m_{i}-1} & 0 \\
-a_{i 0} & 0 & 0 & & 0 & 0 & a_{i m_{i}}
\end{array}\right)
$$

where

$$
a_{i j}=\frac{\left|N_{S \times S}\left(\Delta_{i}^{j}\right)\right|}{\left|P_{i}\right|} .
$$

Direct calculation shows that the determinant of $A_{i}$ is

$$
\operatorname{det}\left(A_{i}\right)=\sum_{j=0}^{m_{i}} \frac{\prod_{k=0}^{m_{i}} a_{i k}}{a_{i j}},
$$

which is nonzero since all the $a_{i j}$ 's are positive integers.

The previous proposition allows us to speak of the characteristic idempotent of a saturated fusion system.

Definition 5.7 For a saturated fusion system $\mathcal{F}$, let $\omega_{\mathcal{F}}$ denote the characteristic idempotent of $\mathcal{F}$.

Remark 5.8 We make the following observations about the proof of Proposition 5.6.

(1) As a byproduct of the proof we have produced an explicit system of equations which we can solve to produce $\omega_{\mathcal{F}}$.

(2) The coefficients in these equations are all integers. Therefore $\omega_{\mathcal{F}}$ can be regarded as an element in the $p$-localized double Burnside ring:

$$
\omega_{\mathcal{F}} \in A(S, S)_{(p)}=A(S, S) \otimes \mathbb{Z}_{(p)} .
$$

(3) The proof actually shows that $\omega_{\mathcal{F}}$ is the unique idempotent in $A(S, S)_{p}^{\wedge}$ (or $\left.A(S, S)_{(p)}\right)$ with the Linckelmann-Webb Properties (a), (b1) and (c). A symmetric argument shows that $\omega_{\mathcal{F}}$ is the unique idempotent in $A(S, S)_{p}^{\wedge}$ with Properties (a), (b2) and (c). We are therefore in the surprising situation that for an idempotent in $A(S, S)_{p}^{\wedge}$ with Properties (a) and (c), the presence of either stability Property (b1) or (b2) implies the presence of the other. 


\section{Stable characteristic idempotents}

In the preceding sections we have undertaken a thorough study of the structure and properties of characteristic idempotents of saturated fusion systems. Going by [7], we will later construct the classifying spectrum of a saturated fusion system $\mathcal{F}$ over a finite $p$-group $S$ as the mapping telescope of the stable characteristic idempotent $\widetilde{\omega}_{\mathcal{F}}$ of $\mathbb{B} S$ induced by the characteristic idempotent $\omega_{\mathcal{F}}$. In this section we briefly study the stable characteristic idempotent and interpret the consequences of the results of the preceding two sections in terms of stable maps, with a particular view towards fusion stability properties as defined below.

Definition 6.1 Let $\mathcal{F}$ be a fusion system over a finite group $S$ and let $X$ be a spectrum. We say a map $f: \mathbb{B} S \rightarrow X$ is right $\mathcal{F}$-stable if for every $P \leq S$ and $\varphi \in \operatorname{Hom}_{\mathcal{F}}(P, S)$ we have

$$
f \circ \mathbb{B} \varphi \simeq f \circ \mathbb{B} \iota_{P} .
$$

Similarly we say that a map $f: X \rightarrow \mathbb{B} S$ is left $\mathcal{F}$-stable if for every $P \leq S$ and $\varphi \in \operatorname{Hom}_{\mathcal{F}}(P, S)$ we have

$$
t r_{\varphi} \circ f \simeq \operatorname{tr}_{P} \circ f
$$

We will often simply say that a map is $\mathcal{F}$-stable. Unless $X \simeq \mathbb{B} S$ in the previous definition, it should be clear from the context whether this means left or right $\mathcal{F}$ stability.

In Section 2 we defined $\widetilde{A}(S, S)_{p}^{\wedge}$ to be the quotient module of $A(S, S)_{p}^{\wedge}$ obtained by quotienting out all trivial basis elements $[P, \psi]$, and discussed the isomorphism

$$
\widetilde{\alpha}: \widetilde{A}(S, S)_{p}^{\wedge} \stackrel{\cong}{\longrightarrow}\{B S, B S\}
$$

of the Segal Conjecture. Since $\widetilde{A}(S, S)_{p}^{\wedge}$ is a free $\mathbb{Z}_{p}^{\wedge}$-module with one basis element $[P, \psi]$ for each conjugacy class of nontrivial $(S, S)$-pairs, we can also regard $\widetilde{A}(S, S)_{p}^{\wedge}$ as a submodule (but not subring) of $A(S, S)_{p}^{\wedge}$ and we do so in this section. From this point of view we define a version of the augmentation $\epsilon$ by

$$
\tilde{\epsilon}:=\epsilon \circ \widetilde{\alpha}^{-1}:\{B S, B S\} \stackrel{\widetilde{\alpha}^{-1}}{\longrightarrow} \tilde{A}(S, S)_{p}^{\wedge} \subseteq A(S, S)_{p}^{\wedge} \stackrel{\epsilon}{\longrightarrow} \mathbb{Z}_{p}^{\wedge}
$$

This is not a ring homomorphism and therefore not an augmentation, although it plays a similar role.

Definition 6.2 Let $\mathcal{F}$ be a fusion system over a finite $p$-group $S$. A stable characteristic idempotent for $\mathcal{F}$ is an idempotent $\omega \in\{B S, B S\}$ with the following properties: 
(a) $\tilde{\omega} \in \tilde{\alpha}\left(A_{\mathcal{F}}(S, S)_{p}^{\wedge}\right)$,

(b1) $\tilde{\omega}$ is right $\mathcal{F}$-stable.

(b2) $\tilde{\omega}$ is left $\mathcal{F}$-stable.

(c) $\tilde{\epsilon}(\widetilde{\omega})=1$.

Proposition 6.3 Every saturated fusion system $\mathcal{F}$ has a unique stable characteristic idempotent $\tilde{\omega}_{\mathcal{F}}$.

Proof If $\mathcal{F}$ is a saturated fusion system over a finite $p$-group $S$, then the image $\tilde{\omega}_{\mathcal{F}}$ in $\{B S, B S\}$ of the characteristic idempotent $\omega_{\mathcal{F}}$ under the surjection

$$
A(S, S)_{p}^{\wedge} \longrightarrow \tilde{A}(S, S)_{p}^{\wedge} \stackrel{\widetilde{\alpha}}{\cong}\{B S, B S\}
$$

is a stable characteristic idempotent for $\mathcal{F}$.

To prove uniqueness, suppose that $\widetilde{\omega}$ is a stable characteristic idempotent of $\mathcal{F}$ and consider $\omega:=\widetilde{\alpha}^{-1}(\widetilde{\omega})$, regarded as an element of $A(S, S)_{p}^{\wedge}$. It is enough to show that $\omega$ is a characteristic idempotent for $\mathcal{F}$, because the result then follows from uniqueness of characteristic idempotents (Proposition 5.6).

It is immediate that $\omega \in A_{\mathcal{F}}(S, S)_{p}^{\wedge}$ and $\epsilon(\omega)=1$. We show that $\omega$ is idempotent. Indeed, idempotence of $\tilde{\omega}$ implies that

$$
v:=\omega \circ \omega-\omega
$$

is a linear combination of trivial basis elements. But since $v$ is an element of $A_{\mathcal{F}}(S, S)_{p}^{\wedge}$, and the only trivial basis element in $A_{\mathcal{F}}(S, S)_{p}^{\wedge}$ is $[1$, triv $]$, where 1 denotes the trivial subgroup, $v$ must be of the form $v=a \cdot[1$, triv $]$. Applying $\epsilon$ we get

$$
1=\epsilon(\omega \circ \omega)=\epsilon(\omega+\nu)=1+a \cdot|S| .
$$

We conclude that $a=0$, whence $v=0$ and $\omega$ is idempotent.

It only remains to prove that $\omega$ has Property (b). This is deduced from Property (b) for $\widetilde{\omega}$ in a similar way.

As a consequence of Lemma 5.5 we now show that stable characteristic idempotents completely characterize fusion-stable maps.

Corollary 6.4 Let $\mathcal{F}$ be a saturated fusion system over a finite $p$-group $S$ and let $X$ be a spectrum. A map $f: \mathbb{B} S \rightarrow X$ is $\mathcal{F}$-stable if and only if

$$
f \circ \tilde{\omega}_{\mathcal{F}} \simeq f
$$

Algebraic 83 Geometric Topology, Volume 6 (2006) 
Similarly, a map $f: X \rightarrow \mathbb{B} S$ is $\mathcal{F}$-stable if and only if

$$
\tilde{\omega}_{\mathcal{F}} \circ f \simeq f
$$

Proof Suppose first that $f: \mathbb{B} S \rightarrow X$ is $\mathcal{F}$-stable. Note that we can write

$$
\omega_{\mathcal{F}} \simeq \sum_{[P] \lesssim[S]}\left(\sum_{[P, \varphi]} \chi_{\left(\mathcal{F}_{S}, \mathcal{F}\right)} \chi_{[P, \varphi]}\left(\omega_{\mathcal{F}}\right)[P, \varphi]\right)
$$

Now,

$$
\begin{aligned}
& f \circ \tilde{\omega}_{\mathcal{F}} \simeq f \circ \sum_{[P] \lesssim[S]}\left(\sum_{[P, \varphi]} \chi_{\left(\mathcal{F}_{S}, \mathcal{F}\right)} \chi_{[P, \varphi]}\left(\omega_{\mathcal{F}}\right) \cdot \tilde{\alpha}([P, \varphi])\right)
\end{aligned}
$$

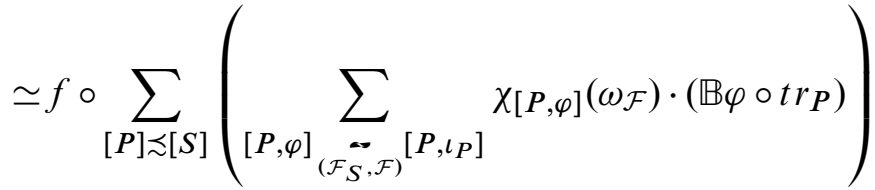

$$
\begin{aligned}
& \simeq \sum_{[P] \lesssim[S]}\left(\sum_{[P, \varphi]} \chi_{\left(\mathcal{F}, \mathcal{F}^{\prime},[P, \varphi]\right.}\left(\omega_{\mathcal{F}}\right) \cdot\left(f \circ \mathbb{B} \varphi \circ t r_{P}\right)\right) \\
& \simeq \sum_{[P] \lesssim[S]}\left(\left(\sum_{[P, \varphi]} \sum_{\left(\mathcal{F}_{S}, \mathcal{F}\right)} \chi_{\left[P, \iota_{P}\right]}\left(\omega_{\mathcal{F}}\right)\right) \cdot\left(f \circ \mathbb{B} \iota_{P} \circ t r_{P}\right)\right) \\
& \simeq \sum_{[P] \lesssim[S]}\left(\chi_{[P]}^{\mathcal{F}}\left(\omega_{\mathcal{F}}\right) \cdot\left(f \circ \mathbb{B} \iota_{P} \circ t r_{P}\right)\right) \text {. }
\end{aligned}
$$

By Lemma 5.5, we have

$$
\chi_{[P]}^{\mathcal{F}}\left(\omega_{\mathcal{F}}\right)= \begin{cases}1 & \text { if } P=S, \\ 0 & \text { if } P<S .\end{cases}
$$

We conclude that

$$
f \circ \tilde{\omega}_{\mathcal{F}} \simeq f \circ \mathbb{B} \iota_{S} \circ t r_{S} \simeq f .
$$

The converse implication follows from $\mathcal{F}$-stability of $\widetilde{\omega}_{\mathcal{F}}$. 
The result for left $\mathcal{F}$-stable maps is deduced similarly by writing

$$
\omega_{\mathcal{F}}=\sum_{[P] \lesssim[S]}\left(\sum_{[Q, \varphi]} \chi_{\left(\mathcal{F}, \mathcal{F}_{S}\right)} \chi_{\left[Q, \iota_{P}\right]}\left(\omega_{\mathcal{F}}\right)[Q, \varphi]\right) .
$$

If $f$ is a left $\mathcal{F}$-stable map $f$, we get the following after some manipulations

$$
\tilde{\omega}_{\mathcal{F}} \circ f \simeq \sum_{[P] \precsim[S]}\left(\chi_{\mathcal{F}}^{[P]}\left(\omega_{\mathcal{F}}\right) \cdot\left(\mathbb{B} \iota_{P} \circ t r_{P} \circ f\right)\right)
$$

and $\tilde{\omega}_{\mathcal{F}} \circ f \simeq f$ follows after appealing to Lemma 5.5.

\section{Classifying spectra}

In this section we develop the theory of classifying spectra for saturated fusion systems. The classifying spectra constructed here agree with those suggested in [7], but the added rigidity of the new construction allows us to prove that the assignment is functorial for fusion-preserving homomorphisms between fusion systems. We will also endow the classifying spectrum of a saturated fusion system $\mathcal{F}$ over a finite $p$-group $S$ with additional structure by regarding it as an object under $\mathbb{B} S$. We support this idea by proving that we can reconstruct the fusion system $\mathcal{F}$ from its classifying spectrum, when regarded as an object under $\mathbb{B} S$.

Let $\mathcal{F}$ be a saturated fusion system over a finite $p$-group $S$ and denote the infinite mapping telescope of the stable characteristic idempotent $\tilde{\omega}_{\mathcal{F}}$ by $\mathbb{B} \mathcal{F}$. In other words,

$$
\mathbb{B F}:=\underset{\operatorname{HoColim}}{\longrightarrow}\left(\mathbb{B} S \stackrel{\widetilde{\omega}_{\mathcal{F}}}{\longrightarrow} \mathbb{B} S \stackrel{\widetilde{\omega}_{\mathcal{F}}}{\longrightarrow} \mathbb{B} S \stackrel{\widetilde{\omega}_{\mathcal{F}}}{\longrightarrow} \ldots\right) .
$$

We denote the structure map of the homotopy colimit by $\sigma_{\mathcal{F}}: \mathbb{B} S \rightarrow \mathbb{B} \mathcal{F}$. Since $\tilde{\omega}_{\mathcal{F}}$ is idempotent up to homotopy, we get a homotopy factorization of $\widetilde{\omega}_{\mathcal{F}}$ through the homotopy colimit

$$
\tilde{\omega}_{\mathcal{F}}: \mathbb{B} S \stackrel{\sigma_{\mathcal{F}}}{\longrightarrow} \mathbb{B} \mathcal{F} \stackrel{t_{\mathcal{F}}}{\longrightarrow} \mathbb{B} S,
$$

such that $\sigma_{\mathcal{F}} \circ t_{\mathcal{F}} \simeq i d_{\mathbb{B} \mathcal{F}}$. Note that $t_{\mathcal{F}}$ is, up to homotopy, the unique map with these properties. Since $\mathbb{B} \mathcal{F}$ is a retract of the $p$-complete spectrum $\mathbb{B} S$, it is $p$-complete.

Definition 7.1 Let $\mathcal{F}$ be a saturated fusion system over a finite $p$-group $S$. The classifying spectrum of $\mathcal{F}$ is the spectrum $\mathbb{B} \mathcal{F}$, the structure map of $\mathcal{F}$ is the map $\sigma_{\mathcal{F}}$, and the transfer of $\mathcal{F}$ is the map

$$
t_{\mathcal{F}}: \mathbb{B} \mathcal{F} \rightarrow \mathbb{B} S
$$

Algebraic 83 Geometric Topology, Volume 6 (2006) 
such that $\sigma_{\mathcal{F}} \circ t_{\mathcal{F}} \simeq i d_{\mathbb{B} \mathcal{F}}$ and $t_{\mathcal{F}} \circ \sigma_{\mathcal{F}} \simeq \tilde{\omega}_{\mathcal{F}}$.

The structured classifying spectrum of $\mathcal{F}$ is the mapping telescope

$$
\sigma_{\mathcal{F}}: \mathbb{B} S \rightarrow \mathbb{B} \mathcal{F}
$$

regarded as an object under $\mathbb{B} S$.

In the special case when $\mathcal{F}=\mathcal{F}_{S}$ is the fusion system of $S$, the stable characteristic idempotent $\widetilde{\omega}_{\mathcal{F}_{S}}$ is just the identity of $\mathbb{B} S$, so the structured classifying spectrum of $\mathcal{F}_{S}$ becomes a natural homotopy equivalence

$$
\sigma_{\mathcal{F}_{S}}: \mathbb{B} S \stackrel{\simeq}{\longrightarrow} \mathbb{B} \mathcal{F}_{S} .
$$

We will therefore often replace $\mathbb{B} \mathcal{F}_{S}$ by $\mathbb{B} S$.

It has been shown in [7] that for a $p$-local finite group $(S, \mathcal{F}, \mathcal{L})$, the classifying spectrum $\mathbb{B} \mathcal{F}$ is homotopy equivalent to the infinite suspension spectrum of the classifying space $|\mathcal{L}|_{p}^{\wedge}$, thus partly justifying the use of the term "classifying spectrum". In Section 10 we extend this observation to structured classifying spectra.

As an obvious consequence of Proposition 5.2, the group of homotopy classes of maps between classifying spectra of fusion systems has an appealingly simple description, analogous to Theorem 2.4. This is Theorem B of the introduction.

Theorem 7.2 Let $\mathcal{F}_{1}$ and $\mathcal{F}_{2}$ be saturated fusion systems over the finite $p$-groups $S_{1}$ and $S_{2}$, respectively, and let $\widetilde{I}$ be the set of $\left(\mathcal{F}_{1}, \mathcal{F}_{2}\right)$-conjugacy classes of nontrivial $\left(S_{1}, S_{2}\right)$-pairs. Pick a representative $\left(P_{i}, \psi_{i}\right)$ for each $i \in \widetilde{I}$. Then the collection

$$
\left\{\sigma_{\mathcal{F}_{2}} \circ \tilde{\alpha}\left(\left[P_{i}, \psi_{i}\right]\right) \circ t_{\mathcal{F}_{1}} \mid i \in \tilde{I}\right\}
$$

forms a $\mathbb{Z}_{p}^{\wedge}$-basis for $\left[\mathbb{B} \mathcal{F}_{1}, \mathbb{B} \mathcal{F}_{2}\right]$.

Proof The map

$$
\left[\mathbb{B} \mathcal{F}_{1}, \mathbb{B} \mathcal{F}_{2}\right] \longrightarrow\left\{B S_{1}, B S_{2}\right\}, \quad f \mapsto t_{\mathcal{F}_{2}} \circ f \circ \sigma_{\mathcal{F}_{1}}
$$

and its left inverse

$$
\left\{B S_{1}, B S_{2}\right\} \longrightarrow\left[\mathbb{B} \mathcal{F}_{1}, \mathbb{B} \mathcal{F}_{2}\right], \quad g \mapsto \sigma_{\mathcal{F}_{2}} \circ g \circ t_{\mathcal{F}_{1}}
$$

make $\left[\mathbb{B} \mathcal{F}_{1}, \mathbb{B} \mathcal{F}_{2}\right]$ isomorphic to the submodule

$$
\widetilde{\omega}_{\mathcal{F}_{2}} \circ\left\{B S_{1}, B S_{2}\right\} \circ \widetilde{\omega}_{\mathcal{F}_{1}} \subset\left\{B S_{1}, B S_{2}\right\} .
$$

Since

$$
\tilde{\omega}_{\mathcal{F}_{2}} \circ\left\{B S_{1}, B S_{2}\right\} \circ \tilde{\omega}_{\mathcal{F}_{1}}=\tilde{\alpha}\left(\omega_{\mathcal{F}_{2}} \circ \tilde{A}\left(S_{1}, S_{2}\right)_{p}^{\wedge} \circ \omega_{\mathcal{F}_{1}}\right),
$$


the result now follows from the explicit description of the $\mathbb{Z}_{p}^{\wedge}$-basis of $\omega_{\mathcal{F}_{2}} \circ A\left(S_{1}, S_{2}\right)_{p}^{\wedge} \circ \omega_{\mathcal{F}_{1}}$ given in Proposition 5.2.

This theorem can be applied to fusion systems arising from finite groups to give a new and simple description of the group of homotopy classes of stable maps between $p$-completed classifying spaces of finite groups. Since this result is of independent interest, it will be presented separately in [24]. The proof given there is similar but more direct, with the added advantage that the double coset formula is preserved, and that the target can be the classifying space of a compact Lie group.

The following theorem further justifies the use of the term classifying spectrum. This appears in the introduction as Theorem A.

Theorem 7.3 If $\mathcal{F}$ is a saturated fusion system over a finite $p$-group $S$, then

$$
\operatorname{Hom}_{\mathcal{F}}(P, Q)=\left\{\varphi \in \operatorname{Hom}(P, Q) \mid \sigma_{\mathcal{F}} \circ \mathbb{B} \iota_{Q} \circ \mathbb{B} \varphi \simeq \sigma_{\mathcal{F}} \circ \mathbb{B} \iota_{P}\right\}
$$

for all subgroups $P, Q \leq S$.

Proof When $P$ is the trivial subgroup there is nothing to show, so we assume otherwise. Now, let $\varphi \in \operatorname{Hom}(P, Q)$. Since $t_{\mathcal{F}} \circ \sigma_{\mathcal{F}} \simeq \widetilde{\omega}_{\mathcal{F}}$ and $\sigma_{\mathcal{F}} \circ \widetilde{\omega}_{\mathcal{F}} \simeq \sigma_{\mathcal{F}}$, we have

$$
\sigma_{\mathcal{F}} \circ \mathbb{B} \iota_{Q} \circ \mathbb{B} \varphi \simeq \sigma_{\mathcal{F}} \circ \mathbb{B}_{P}
$$

if and only if

$$
\tilde{\omega}_{\mathcal{F}} \circ \mathbb{B} \iota_{Q} \circ \mathbb{B} \varphi \simeq \tilde{\omega}_{\mathcal{F}} \circ \mathbb{B} \iota_{P},
$$

which we rewrite as

$$
\tilde{\omega}_{\mathcal{F}} \circ \widetilde{\alpha}\left(\left[P, \iota_{Q} \circ \varphi\right]_{P}^{S}\right) \simeq \tilde{\omega}_{\mathcal{F}} \circ \widetilde{\alpha}\left(\left[P, \iota_{P}\right]_{P}^{S}\right) .
$$

Since $\mathbb{B} P$ is naturally equivalent to the classifying spectrum of $\mathcal{F}_{P}$, the fusion system of $P$, we can apply Theorem 7.2 to see that the last equivalence holds if and only if the $(P, S)$-pairs $\left(P, \iota_{Q} \circ \varphi\right)$ and $\left(P, \iota_{P}\right)$ are $\left(\mathcal{F}_{P}, \mathcal{F}\right)$-conjugate. By definition this means that there exist $g \in P$ and $\varphi^{\prime} \in \operatorname{Hom}_{\mathcal{F}}(P, \varphi(P))$ such that the following diagram commutes

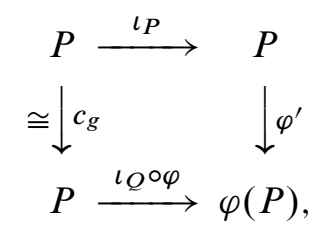

or in other words such that

$$
\varphi(x)=\varphi^{\prime} \circ c_{g}^{-1}(x)
$$

for all $x \in P$. This is in turn true if and only $\varphi \in \operatorname{Hom}_{\mathcal{F}}(P, Q)$. 
This theorem shows in particular that the fusion system of a finite group $G$ with Sylow subgroup $S$ is determined by $\mathbb{B} G$ regarded as an object under $\mathbb{B} S$. Example 5.2 of [14] shows that the homotopy type of $\mathbb{B} G$ alone does not determine the fusion system; the classifying spectrum must be regarded as an object under $\mathbb{B} S$. As this result is also of independent interest, and can be proved directly using the Segal Conjecture, it is treated separately in [22].

We will show that the assignment of a classifying spectrum to a saturated fusion systems is functorial, but first we need to specify which notion of morphisms between fusion systems we are working with. The following definition appeared in [21] but using different terminology.

Definition 7.4 Let $\mathcal{F}_{1}$ and $\mathcal{F}_{2}$ be fusion systems over finite $p$-groups $S_{1}$ and $S_{2}$, respectively. A $\left(\mathcal{F}_{1}, \mathcal{F}_{2}\right)$-fusion-preserving homomorphism is a group homomorphism $\gamma: S_{1} \longrightarrow S_{2}$ for which there exists a functor $F_{\gamma}: \mathcal{F}_{1} \longrightarrow \mathcal{F}_{2}$ such that

$$
F_{\gamma}(P)=\gamma(P)
$$

for all subgroups $P \leq S_{1}$, and

$$
\left.\gamma\right|_{Q} \circ \varphi=\left.F_{\gamma}(\varphi) \circ \gamma\right|_{P}
$$

for all $\varphi \in \operatorname{Hom}_{\mathcal{F}_{1}}(P, Q)$.

Remark 7.5 When there is no danger of confusion, we will often say simply that $\gamma$ is fusion-preserving. The functor $F_{\gamma}$ is uniquely determined by the fusion-preserving morphism $\gamma$ in the above definition and we will from now on let $F_{\gamma}$ denote the functor defined by a fusion-preserving homomorphism $\gamma$.

As a motivation for this definition one may may consider the equation $\gamma \circ c_{g}(x)=c_{\gamma(g)} \circ \gamma(x)$ for a group homomorphism $\gamma: G \rightarrow H$ and elements $g, x \in G$. Certainly it follows easily from this equation that a homomorphism between finite groups induces a fusion-preserving homomorphism between their fusion systems. Similarly it is not too difficult to prove that a map between classifying spaces of $p$-local finite groups induces a fusion-preserving homomorphism between their fusion systems (see Remark 7.12).

Definition 7.6 Let FS be the category whose objects are pairs $(S, \mathcal{F})$ consisting of a finite $p$-group $S$ and a fusion system $\mathcal{F}$ over $S$, and whose morphisms are fusionpreserving homomorphisms. Let SFS be the full subcategory of FS whose objects are pairs $(S, \mathcal{F})$ where $\mathcal{F}$ is saturated. 
Since a given homomorphism $\gamma: S_{1} \rightarrow S_{2}$ can be fusion-preserving for many different fusion systems, we will write $\gamma_{\mathcal{F}_{1}}^{\mathcal{F}_{2}}$ to specify that it is regarded as an element in the morphism set $\operatorname{Mor}_{\mathrm{FS}}\left(\left(S_{1}, \mathcal{F}_{1}\right),\left(S_{2}, \mathcal{F}_{2}\right)\right)$.

Lemma 7.7 Let $\mathcal{F}_{1}$ and $\mathcal{F}_{2}$ be saturated fusion systems over finite $p$-groups $S_{1}$ and $S_{2}$, respectively, and let $\gamma: S_{1} \rightarrow S_{2}$ be a fusion-preserving homomorphism. If $f: \mathbb{B} S_{2} \rightarrow X$ is a $\mathcal{F}_{2}$-stable map then

$$
f \circ \mathbb{B} \gamma: \mathbb{B} S_{1} \rightarrow X
$$

is $\mathcal{F}_{1}$-stable, and

$$
f \circ \mathbb{B} \gamma \circ \tilde{\omega}_{\mathcal{F}_{1}} \simeq f \circ \mathbb{B} \gamma
$$

Proof Indeed, for $P \leq S_{1}$ and $\varphi \in \operatorname{Hom}_{\mathcal{F}_{1}}\left(P, S_{1}\right)$ we have

$$
\begin{aligned}
f \circ \mathbb{B} \gamma \circ \mathbb{B} \varphi & \simeq f \circ \mathbb{B}(\gamma \circ \varphi) \\
& \left.\simeq f \circ \mathbb{B} F_{\gamma}(\varphi) \circ \mathbb{B} \gamma\right|_{P} \\
& \left.\simeq f \circ \mathbb{B} \iota_{\gamma(P)} \circ \mathbb{B} \gamma\right|_{P} \\
& \simeq f \circ \mathbb{B}\left(\gamma \circ \iota_{P}\right) \\
& \simeq f \circ \mathbb{B} \gamma \circ \mathbb{B} \iota_{P},
\end{aligned}
$$

proving $\mathcal{F}_{1}$-stability. The second claim follows from Corollary 6.4.

Now, given saturated fusion systems $\mathcal{F}_{1}$ and $\mathcal{F}_{2}$ over finite $p$-groups $S_{1}$ and $S_{2}$, respectively, and a fusion-preserving homomorphism $\gamma: S_{1} \rightarrow S_{2}$, we define a map of spectra

$$
\mathbb{B} \gamma_{\mathcal{F}_{1}}^{\mathcal{F}_{2}}: \mathbb{B} \mathcal{F}_{1} \rightarrow \mathbb{B} \mathcal{F}_{2}
$$

by

$$
\mathbb{B} \gamma_{\mathcal{F}_{1}}^{\mathcal{F}_{2}}:=\sigma_{\mathcal{F}_{2}} \circ \mathbb{B} \gamma \circ t_{\mathcal{F}_{1}}
$$

We will show that this assignment is functorial below. The proof will first be presented in a setting where the target category keeps track of the structure maps of the classifying spectra. To this end we make the following definition.

Definition 7.8 Let $\mathcal{C}$ be the category whose objects are maps of spectra

$$
\sigma: \mathbb{B} S \rightarrow X,
$$

where $S$ is a finite $p$-group and $X$ is a $p$-complete spectrum, and where the set of morphisms from $\sigma_{1}: \mathbb{B} S_{1} \rightarrow X_{1}$ to $\sigma_{2}: \mathbb{B} S_{2} \rightarrow X_{2}$ consists of pairs $(\gamma, g)$, where 
$\gamma: S_{1} \rightarrow S_{2}$ is a group homomorphism and $g: X_{1} \rightarrow X_{2}$ is a map of spectra, such that the following diagram commutes up to homotopy:

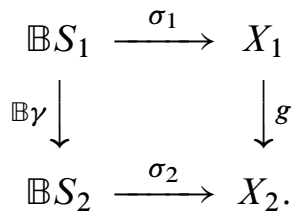

Theorem 7.9 There is a structured classifying spectrum functor

$$
\Upsilon: S F S \longrightarrow \mathcal{C}
$$

defined on objects by

$$
(S, \mathcal{F}) \mapsto\left(\sigma_{\mathcal{F}}: \mathbb{B} S \rightarrow \mathbb{B} \mathcal{F}\right)
$$

and on morphisms by

$$
\gamma_{\mathcal{F}_{1}}^{\mathcal{F}_{2}} \mapsto\left(\gamma, \mathbb{B} \gamma_{\mathcal{F}_{1}}^{\mathcal{F}_{2}}\right)
$$

Proof There are three things to show. First, it is clear by construction that $\sigma_{\mathcal{F}}: \mathbb{B} S \rightarrow \mathbb{B} \mathcal{F}$ is an object of $\mathcal{C}$.

Second, if $\gamma_{\mathcal{F}_{1}}^{\mathcal{F}_{2}} \in \operatorname{Mor} \operatorname{SFS}\left(\left(S_{1}, \mathcal{F}_{1}\right),\left(S_{2}, \mathcal{F}_{2}\right)\right)$, then we need to prove commutativity of the diagram

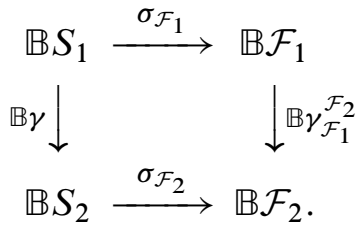

Going around the top half of the square, we get

$$
\begin{aligned}
\mathbb{B} \gamma_{\mathcal{F}_{1}}^{\mathcal{F}_{2}} \circ \sigma_{\mathcal{F}_{1}} & =\sigma_{\mathcal{F}_{2}} \circ \mathbb{B} \gamma \circ t_{\mathcal{F}_{1}} \circ \sigma_{\mathcal{F}_{1}} \\
& \simeq \sigma_{\mathcal{F}_{2}} \circ \mathbb{B} \gamma \circ \tilde{\omega}_{\mathcal{F}_{1}} .
\end{aligned}
$$

Going around the bottom half of the square, we get

$$
\sigma_{\mathcal{F}_{2}} \circ \mathbb{B} \gamma .
$$

Since $\sigma_{\mathcal{F}_{2}}$ is $\mathcal{F}_{2}$-stable, these are the same by Lemma 7.7.

Third, we need to show that $\Upsilon$ preserves compositions. For this, we let $\left(S_{1}, \mathcal{F}_{1}\right),\left(S_{2}, \mathcal{F}_{2}\right)$ and $\left(S_{3}, \mathcal{F}_{3}\right)$ be saturated fusion systems, and

$$
\gamma_{1}: S_{1} \rightarrow S_{2} \quad \text { and } \quad \gamma_{2}: S_{2} \rightarrow S_{3}
$$


be fusion-preserving morphisms between them. Now,

$$
\begin{aligned}
\mathbb{B} \gamma_{2} \mathcal{F}_{\mathcal{F}_{2}}^{\mathcal{F}_{3}} \circ \mathbb{B} \gamma_{1} \mathcal{F}_{\mathcal{F}_{1}}^{\mathcal{F}_{2}} & \simeq \sigma_{\mathcal{F}_{3}} \circ \mathbb{B} \gamma_{2} \circ \underbrace{t_{\mathcal{F}_{2}} \circ \sigma_{\mathcal{F}_{2}}}_{=\widetilde{\omega}_{\mathcal{F}_{2}}} \circ \mathbb{B} \gamma_{1} \circ t_{\mathcal{F}_{1}} \\
& \stackrel{(7.7)}{\simeq} \sigma_{\mathcal{F}_{3}} \circ \mathbb{B} \gamma_{2} \circ \mathbb{B} \gamma_{1} \circ t_{\mathcal{F}_{1}} \\
& \simeq \sigma_{\mathcal{F}_{3}} \circ \mathbb{B}\left(\gamma_{2} \circ \gamma_{1}\right) \circ t_{\mathcal{F}_{1}} \\
& \simeq \mathbb{B}\left(\gamma_{2} \circ \gamma_{1}\right)_{\mathcal{F}_{1}}^{\mathcal{F}_{3}}
\end{aligned}
$$

This completes the proof.

Composing with the forgetful functor from $\mathcal{C}$ to Spectra, we obtain the following corollary.

Corollary 7.10 There is a classifying spectrum functor

$$
\mathbb{B}: S F S \longrightarrow \text { Spectra }
$$

defined on objects by

$$
(S, \mathcal{F}) \mapsto \mathbb{B} \mathcal{F}
$$

and on morphisms by

$$
\gamma_{\mathcal{F}_{1}}^{\mathcal{F}_{2}} \mapsto \mathbb{B} \gamma_{\mathcal{F}_{1}}^{\mathcal{F}_{2}}
$$

We conclude this section by illustrating that the fusion-preserving homomorphisms are the only homomorphisms inducing maps between classifying spectra that preserve structure maps.

Proposition 7.11 Let $\mathcal{F}_{1}$ and $\mathcal{F}_{2}$ be saturated fusion systems over finite $p$-groups $S_{1}$ and $S_{2}$, respectively. If $\gamma: S_{1} \rightarrow S_{2}$ is a group homomorphism such that $\mathbb{B} \gamma: \mathbb{B} S_{1} \rightarrow \mathbb{B} S_{2}$ restricts to a map $g: \mathbb{B} \mathcal{F}_{1} \rightarrow \mathbb{B} \mathcal{F}_{2}$ making the following diagram commute up to homotopy

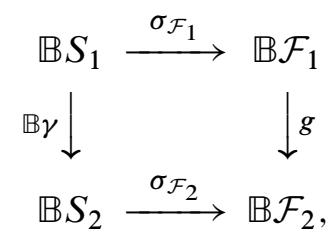

then $\gamma$ is fusion-preserving. 
Proof We will produce a functor $F_{\gamma}: \mathcal{F}_{1} \rightarrow \mathcal{F}_{2}$ that makes $\gamma$ fusion-preserving. There are two things to check. First, that given a homomorphism $\varphi \in \operatorname{Hom}_{\mathcal{F}_{1}}(P, Q)$ there is a unique induced homomorphism $F_{\gamma}(\varphi): \gamma(P) \rightarrow \gamma(Q)$ such that $\left.\gamma\right|_{Q} \circ \varphi=\left.F_{\gamma}(\varphi) \circ \gamma\right|_{P}$, and second, that $F_{\gamma}(\varphi)$ is in $\mathcal{F}_{2}$. Functoriality of $F_{\gamma}$ follows from the uniqueness.

To prove the first claim, let $K$ be the kernel of $\gamma$. Then $K \cap P$ is the kernel of $\left.\gamma\right|_{P}$, and by standard group theory there exists a homomorphism $F_{\gamma}(\varphi)$ fitting into the following commutative diagram

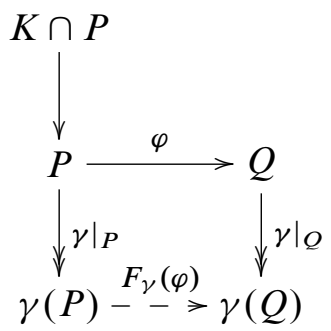

if and only if the restriction of $\left.\gamma\right|_{Q} \circ \varphi$ to $K \cap P$ is trivial. Furthermore, since $\left.\gamma\right|_{P}$ is surjective onto $\gamma(P)$, this condition uniquely determines $F_{\gamma}(\varphi)$ if it exists. Now,

$$
\begin{aligned}
\sigma_{\mathcal{F}_{2}} \circ \mathbb{B}\left(\left.\left.\gamma\right|_{Q} \circ \varphi\right|_{K \cap P}\right) & \simeq \sigma_{\mathcal{F}_{2}} \circ \mathbb{B} \gamma \circ \mathbb{B} \iota_{Q} \circ \mathbb{B} \varphi \circ \mathbb{B} \iota_{K \cap P} \\
& \simeq g \circ \sigma_{\mathcal{F}_{1}} \circ \mathbb{B} \iota_{Q} \circ \mathbb{B} \varphi \circ \mathbb{B} \iota_{K \cap P} \\
& \simeq g \circ \sigma_{\mathcal{F}_{1}} \circ \mathbb{B} \iota_{P} \circ \mathbb{B} \iota_{K \cap P} \\
& \simeq \sigma_{\mathcal{F}_{2}} \circ \mathbb{B} \gamma \circ \mathbb{B} \iota_{K \cap P} \\
& \simeq * .
\end{aligned}
$$

By applying Theorem 7.2 we conclude that $\left(\left.\left.\gamma\right|_{Q} \circ \varphi\right|_{K \cap P}\right)$ is trivial.

The second claim is proved similarly by first performing the following manipulation

$$
\begin{aligned}
\sigma_{\mathcal{F}_{2}} \circ \mathbb{B} \iota_{\gamma}(Q) \circ \mathbb{B} F_{\gamma}(\varphi) \circ \mathbb{B} \gamma \mid P & \left.\simeq \sigma_{\mathcal{F}_{2}} \circ \mathbb{B} \iota_{\gamma(Q)} \circ \mathbb{B} \gamma\right|_{Q} \circ \mathbb{B} \varphi \\
& \simeq g \circ \sigma_{\mathcal{F}_{1}} \circ \mathbb{B} \iota_{Q} \circ \mathbb{B} \varphi \\
& \simeq g \circ \sigma_{\mathcal{F}_{1}} \circ \mathbb{B} \iota_{P} \\
& \simeq \sigma_{\mathcal{F}_{2}} \circ \mathbb{B} \gamma \circ \mathbb{B} \iota_{P},
\end{aligned}
$$

from which we conclude by Theorem 7.2, that the $\left(P, S_{2}\right)$-pair $\left(P,\left.\iota_{\gamma}(Q) \circ F_{\gamma}(\varphi) \circ \gamma\right|_{P}\right)$ is $\left(\mathcal{F}_{P}, \mathcal{F}_{2}\right)$-conjugate to $\left(P, \gamma \circ \iota_{P}\right)$. By definition this means that there exist $g \in P$ and $\varphi^{\prime} \in \operatorname{Hom}_{\mathcal{F}_{2}}(\gamma(P), \gamma(\varphi(P)))$ making the 
following diagram commute

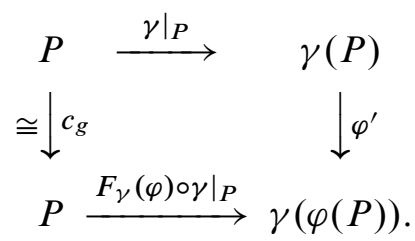

This implies that

$$
\begin{aligned}
F_{\gamma}(\varphi) \circ \gamma(x) & =\varphi^{\prime} \circ \gamma \circ c_{g^{-1}}(x) \\
& =\varphi^{\prime} \circ c_{\gamma\left(g^{-1}\right)} \circ \gamma(x),
\end{aligned}
$$

for all $x \in P$. Since $\gamma$ is surjective onto $\gamma(P)$ this implies that

$$
F_{\gamma}(\varphi)=\varphi^{\prime} \circ c_{\gamma\left(g^{-1}\right)} \in \operatorname{Hom}_{\mathcal{F}_{2}}(\gamma(P), \gamma(Q)) \text {. }
$$

\section{Remark 7.12}

(1) An unstable version of the preceding proof, using [7, Proposition 4.4] instead of Theorem 7.2, shows that a map between classifying spaces of $p$-local finite groups restricts to a fusion-preserving homomorphism of underlying $p$-groups.

(2) In the second paragraph of the proof we showed that

$$
\varphi(\operatorname{Ker} \gamma \cap P) \leq \operatorname{Ker} \gamma
$$

for all $P, Q \leq S$ and $\varphi \in \operatorname{Hom}_{\mathcal{F}_{1}}(P, Q)$. In other words, $\operatorname{Ker} \gamma$ is strongly closed in $\mathcal{F}_{1}$.

\section{Transfer theory for classifying spectra}

In the classical setting, the map of classifying spaces induced by an injective homomorphism $\psi: G \rightarrow H$ of finite groups admits a transfer $t r_{\psi}: \Sigma_{+}^{\infty} B H \rightarrow \Sigma_{+}^{\infty} B G$ (see for example [2]), which restricts to a reduced transfer $\Sigma^{\infty} B H \rightarrow \Sigma^{\infty} B G$, also denoted by $t r_{\psi}$. In this section we adapt this theory to injective fusion-preserving homomorphisms and obtain a transfer theory between classifying spectra. The transfers we construct will not respect structure maps of saturated fusion systems and so do not fit into the framework of structured classifying spectra. They do however respect transfer maps of saturated fusion systems and we will formulate our results in terms of a functor analogous to the structured classifying space functor. One motivation for doing so is the following, rather surprising result. 
Proposition 8.1 If $\mathcal{F}$ is a saturated fusion system over a finite $p$-group $S$, then

$$
\operatorname{Hom}_{\mathcal{F}}(P, Q)=\left\{\varphi \in \operatorname{Inj}(P, Q) \mid t r_{\varphi} \circ t r_{Q} \circ t_{\mathcal{F}} \simeq t r_{P} \circ t_{\mathcal{F}}\right\}
$$

Proof The proof is similar to the proof of Theorem 7.3. The extra condition that $\varphi$ be injective is required to ensure the existence of the transfer $t r_{\varphi}$.

Definition 8.2 Let $\mathcal{F}_{1}$ and $\mathcal{F}_{2}$ be saturated fusion systems over finite $p$-groups $S_{1}$ and $S_{2}$, respectively. If $\gamma$ is an injective, fusion-preserving homomorphism $S_{1} \rightarrow S_{2}$, then the $\left(\mathcal{F}_{1}, \mathcal{F}_{2}\right)$-transfer of $\gamma$ is

$$
\operatorname{Tr}\left(\gamma_{\mathcal{F}_{1}}^{\mathcal{F}_{2}}\right):=\sigma_{\mathcal{F}_{1}} \circ t r_{\gamma} \circ t_{\mathcal{F}_{2}}: \mathbb{B} \mathcal{F}_{2} \rightarrow \mathbb{B} \mathcal{F}_{1}
$$

As in the previous section we show that this assignment is functorial, working first with a target category that keeps track of all the structure involved.

Definition 8.3 Let $\mathrm{SFS}_{0}$ be the subcategory of SFS, whose objects are saturated fusion systems, but whose morphisms are the injective fusion-preserving homomorphisms.

Definition 8.4 Let $\mathcal{D}$ be the category whose objects are maps of spectra

$$
t: X \rightarrow \mathbb{B} S,
$$

where $S$ is a finite $p$-group and $X$ is a $p$-complete spectrum, and where the set of morphisms from $t_{2}: X_{2} \rightarrow \mathbb{B} S_{2}$ to $t_{1}: X_{1} \rightarrow \mathbb{B} S_{1}$ consists of pairs $(\gamma, g)$ where $\gamma: S_{1} \rightarrow S_{2}$ is a group monomorphism and $g: X_{2} \rightarrow X_{1}$ is a map of spectra such that the following diagram commutes up to homotopy:

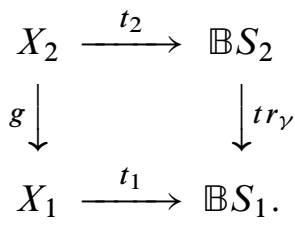

The proof of functoriality for transfers is very similar to the proof of Theorem 7.9 and we will need the following analogue of Lemma 7.7.

Lemma 8.5 Let $\mathcal{F}_{1}$ and $\mathcal{F}_{2}$ be saturated fusion systems over finite $p$-groups $S_{1}$ and $S_{2}$, respectively, and let $\gamma: S_{1} \rightarrow S_{2}$ be a fusion-preserving homomorphism. If $f: X \rightarrow \mathbb{B} S_{2}$ is a $\mathcal{F}_{2}$-stable map, then

$$
t_{\gamma} \circ f: X \rightarrow \mathbb{B} S_{1}
$$

is $\mathcal{F}_{1}-$ stable and

$$
\widetilde{\omega}_{\mathcal{F}_{1}} \circ t r_{\gamma} \circ f=t r_{\gamma} \circ f
$$

Algebraic 83 Geometric Topology, Volume 6 (2006) 
Proof Let $P \leq S_{1}$ and $\varphi \in \operatorname{Hom}_{\mathcal{F}_{1}}(P, S)$. Recalling that the assignment of transfers to group monomorphisms contravariantly preserves compositions up to homotopy, we get

$$
\begin{aligned}
t r_{\varphi} \circ t r_{\gamma} \circ f & \simeq \operatorname{tr}_{(\gamma \circ \varphi)} \circ f \\
& \simeq \operatorname{tr}_{\left(\left.F_{\gamma}(\varphi) \circ \gamma\right|_{P}\right)} \circ f \\
& \simeq t r_{\left.\gamma\right|_{P}} \circ t r_{F_{\gamma}(\varphi)} \circ f \\
& \simeq t r_{\left.\gamma\right|_{P}} \circ t r_{\gamma(P)} \circ f \\
& \simeq t r_{\left(\left.\iota_{\gamma(P)} \circ \gamma\right|_{P}\right)} \circ f \\
& \simeq t r_{\left(\gamma \circ \iota_{P}\right)} \circ f \\
& \simeq t r_{P} \circ t r_{\gamma} \circ f,
\end{aligned}
$$

proving $\mathcal{F}_{1}$-stability. The second claim follows from Corollary 6.4 .

Theorem 8.6 There is a structured fusion transfer functor

$$
\Xi: \mathrm{SFS}_{0} \rightarrow \mathcal{D}^{o p}
$$

defined on objects by

$$
(S, \mathcal{F}) \mapsto\left(t_{\mathcal{F}}: \mathbb{B} \mathcal{F} \rightarrow \mathbb{B} S\right)
$$

and on morphisms by

$$
\gamma_{\mathcal{F}_{1}}^{\mathcal{F}_{2}} \mapsto\left(\gamma, \operatorname{Tr}\left(\gamma_{\mathcal{F}_{1}}^{\mathcal{F}_{2}}\right)\right)
$$

Proof The proof is similar to the proof of Theorem 7.9. There are again three things to show. First, it is clear by construction that $t_{\mathcal{F}}: \mathbb{B} \mathcal{F} \rightarrow \mathbb{B} S$ is an object of $\mathcal{D}$.

Second, if $\gamma_{\mathcal{F}_{1}}^{\mathcal{F}_{2}} \in \operatorname{Mor}_{\mathrm{SFS}_{0}}\left(\left(S_{1}, \mathcal{F}_{1}\right),\left(S_{2}, \mathcal{F}_{2}\right)\right)$, then we prove commutativity of the diagram

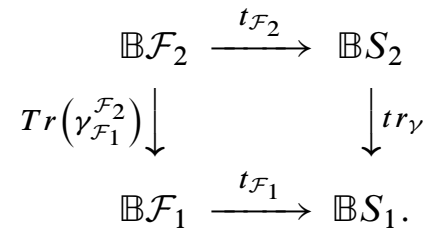

by applying Lemma 8.5 and recalling that $t_{\mathcal{F}_{2}}$ is $\mathcal{F}_{2}$-stable (since $\widetilde{\omega}_{\mathcal{F}_{2}}$ is $\mathcal{F}_{2}$-stable).

The third part, that $\Xi$ preserves compositions, also follows from Lemma 8.5 and functoriality of the classical transfer, just as in the proof of Theorem 7.9.

Composing with the forgetful functor from $\mathcal{D}^{o p}$ to Spectra ${ }^{o p}$ we obtain the following corollary. 
Corollary 8.7 There is a fusion transfer functor

$$
\mathrm{SFS}_{0} \rightarrow \text { Spectra }^{o p}
$$

defined on objects by

$$
(S, \mathcal{F}) \mapsto \mathbb{B} \mathcal{F}
$$

and on morphisms by

$$
\gamma_{\mathcal{F}_{1}}^{\mathcal{F}_{2}} \mapsto \operatorname{Tr}\left(\gamma_{\mathcal{F}_{1}}^{\mathcal{F}_{2}}\right)
$$

We conclude this section with an application of the transfer theory. If $\mathcal{F}_{1}$ and $\mathcal{F}_{2}$ are saturated fusion systems over a finite $p$-group $S$, then we say that a map

$$
f: \mathbb{B} \mathcal{F}_{2} \rightarrow \mathbb{B} \mathcal{F}_{1}
$$

is structure-preserving if $f \circ \sigma_{\mathcal{F}_{2}} \simeq \sigma_{\mathcal{F}_{1}}$. We say that $\mathbb{B} \mathcal{F}_{2}$ is a summand of $\mathbb{B} \mathcal{F}_{1}$ as objects under $\mathbb{B} S$ if there is a structure-preserving map $f: \mathbb{B} \mathcal{F}_{1} \rightarrow \mathbb{B} \mathcal{F}_{2}$ and a map $r: \mathbb{B} \mathcal{F}_{2} \rightarrow \mathbb{B} \mathcal{F}_{1}$ such that

$$
f \circ r \simeq i d_{\mathbb{B} \mathcal{F}_{2}} \text {. }
$$

Proposition 8.8 Let $\mathcal{F}_{1}$ and $\mathcal{F}_{2}$ be saturated fusion systems over a finite $p$-group $S$. The following four conditions are equivalent:

(i) $\mathcal{F}_{1}$ is a subcategory of $\mathcal{F}_{2}$;

(ii) $i d_{S}$ is $\left(\mathcal{F}_{1}, \mathcal{F}_{2}\right)$-fusion-preserving;

(iii) $\mathbb{B} \mathcal{F}_{2}$ is a summand of $\mathbb{B} \mathcal{F}_{1}$ as objects under $\mathbb{B} S$;

(iv) There is a structure-preserving map $f: \mathbb{B} \mathcal{F}_{1} \rightarrow \mathbb{B} \mathcal{F}_{2}$.

Proof (i) $\Rightarrow$ (ii): Obvious.

(ii) $\Rightarrow$ (iii): The map $\mathbb{B} i d_{\mathcal{F}_{1}}^{\mathcal{F}_{2}}$ is structure-preserving and

$$
\begin{aligned}
\mathbb{B i} d_{\mathcal{F}_{1}}^{\mathcal{F}_{2}} \circ \operatorname{Tr}\left(i d_{\mathcal{F}_{1}}^{\mathcal{F}_{2}}\right) & =\sigma_{\mathcal{F}_{2}} \circ \mathbb{B} i d \circ t_{\mathcal{F}_{1}} \circ \sigma_{\mathcal{F}_{1}} \circ t r_{i d} \circ t_{\mathcal{F}_{2}} \\
& =\sigma_{\mathcal{F}_{2}} \circ i d \circ \tilde{\omega}_{\mathcal{F}_{1}} \circ i d \circ t_{\mathcal{F}_{2}} \\
& \stackrel{(7.7)}{=} \sigma_{\mathcal{F}_{2}} \circ t_{\mathcal{F}_{2}} \\
& =i d_{\mathbb{B} \mathcal{F}_{2}} .
\end{aligned}
$$

(iii) $\Rightarrow$ (iv): Obvious.

(iv) $\Rightarrow$ (i): Let $P \leq S$ and $\varphi \in \operatorname{Hom}_{\mathcal{F}_{1}}(P, S)$. Then

$$
\sigma_{\mathcal{F}_{1}} \circ \mathbb{B} \varphi \simeq \sigma_{\mathcal{F}_{1}} \circ \mathbb{B} \iota_{P} .
$$

Algebraic $\mathcal{E} \mathcal{G}$ Geometric Topology, Volume 6 (2006) 
Consequently

$$
\sigma_{\mathcal{F}_{2}} \circ \mathbb{B} \varphi \simeq f \circ \sigma_{\mathcal{F}_{1}} \circ \mathbb{B} \varphi \simeq f \circ \sigma_{\mathcal{F}_{1}} \circ \mathbb{B} \iota_{P} \simeq \sigma_{\mathcal{F}_{2}} \circ \mathbb{B} \iota_{P} .
$$

By Theorem 7.3 it follows that $\varphi \in \operatorname{Hom}_{\mathcal{F}_{2}}(P, S)$.

\section{Cohomology of classifying spectra}

In this section we apply the $\bmod p$ cohomology functor to the theory of the previous sections and observe that the cohomology of fusion systems and their classifying spectra behaves like group cohomology in many important ways. Cohomology will always be taken with $\mathbb{F}_{p}$-coefficients and therefore we will denote the functor $H^{*}\left(-; \mathbb{F}_{p}\right)$ by $H^{*}(-)$.

We begin by recording the analogue of the last part of Proposition 4.2

Proposition 9.1 Let $\mathcal{F}$ be a saturated fusion system over a finite $p$-group $S$. Then the map $\widetilde{\omega}_{\mathcal{F}}^{*}$ induced by its stable characteristic idempotent in cohomology is an idempotent in End $\left(H^{*}(B S)\right)$, is $H^{*}(\mathcal{F})$-linear and a homomorphism of modules over the Steenrod algebra; and

$$
\operatorname{Im}\left[H^{*}(B S) \stackrel{\widetilde{\omega}_{\mathcal{F}}^{*}}{\longrightarrow} H^{*}(B S)\right]=H^{*}(\mathcal{F}) .
$$

Proof The proof is essentially the same as in [7]. The reader is referred to [7, Proposition 5.5] for details.

The next proposition, which is arguably the most important result in this section, has already been observed by Broto-Levi-Oliver in [7].

Proposition 9.2 [7, Section 5] Let $\mathcal{F}$ be a saturated fusion system over a finite $p$-group $S$. Then the structure map $\sigma_{\mathcal{F}}$ induces a split monomorphism

$$
H^{*}(\mathbb{B} \mathcal{F}) \hookrightarrow H^{*}(B S)
$$

in cohomology, with image $H^{*}(\mathcal{F})$.

Proof By construction it is clear that $\sigma_{\mathcal{F}}$ induces the inclusion

$$
\operatorname{Im}\left[H^{*}(B S) \stackrel{\widetilde{\omega}_{\mathcal{F}}^{*}}{\longrightarrow} H^{*}(B S)\right] \hookrightarrow H^{*}(B S)
$$

in cohomology, and by Proposition 9.1 this has image $H^{*}(\mathcal{F})$. 
Remark 9.3 Using Corollary 6.4, Castellana-Morales show in [9] that the analogous result holds for any generalized cohomology theory. That is, for a saturated fusion system $\mathcal{F}$ over a finite $p$-group $S$ and a generalized cohomology theory $E$, the structure map $\sigma_{\mathcal{F}}$ induces a split monomorphism

$$
E^{*}(\mathbb{B} \mathcal{F}) \hookrightarrow E^{*}(B S)
$$

with image

$$
E^{*}(\mathcal{F}):=\varliminf_{\mathcal{F}} E^{*}(B(-)) \text {. }
$$

Since $H^{*}(\mathcal{F})$ is a subring of $H^{*}(B S)$, and is consequently an unstable algebra over the Steenrod algebra, we can now regard $H^{*}(\mathbb{B} \mathcal{F})$ as an unstable algebra over the Steenrod algebra via the isomorphism

$$
\sigma_{\mathcal{F}}^{*}: H^{*}(\mathbb{B} \mathcal{F}) \stackrel{\cong}{\longrightarrow} H^{*}(\mathcal{F}) .
$$

With this understanding, the map

$$
\sigma_{\mathcal{F}}^{*}: H^{*}(\mathbb{B} \mathcal{F}) \longrightarrow H^{*}(\mathbb{B} S)
$$

is obviously a map of unstable algebras over the Steenrod algebra.

The $H^{*}(\mathcal{F})$-linearity of Proposition 9.1 is a version of what is commonly referred to as Frobenius reciprocity in the classical group cohomology setting. We record this property in the more familiar form.

Proposition 9.4 (Frobenius reciprocity) Let $\mathcal{F}$ be a saturated fusion system over a finite $p$-group $S$. If $x \in H^{*}(\mathbb{B} \mathcal{F})$ and $y \in H^{*}(B S)$, then

$$
t_{\mathcal{F}}^{*}\left(\sigma_{\mathcal{F}}^{*}(x) \cdot y\right)=x \cdot t_{\mathcal{F}}^{*}(y) .
$$

Proof Since there is only one fusion system in play, we will drop the subscript $\mathcal{F}$ and simply write $\sigma, t$ and $\widetilde{\omega}$. Now, by using $H^{*}(\mathcal{F})$-linearity of $\widetilde{\omega}^{*}$ and the fact that $\sigma^{*}$ preserves multiplication, we have

$$
\begin{aligned}
t^{*}\left(\sigma^{*}(x) \cdot y\right) & =t^{*} \circ \widetilde{\omega}^{*}(\underbrace{\sigma^{*}(x)}_{\in H^{*}(\mathcal{F})} \cdot y) \\
& =t^{*}\left(\sigma^{*}(x) \cdot \widetilde{\omega}^{*}(y)\right) \\
& =t^{*}\left(\sigma^{*}(x) \cdot\left(\sigma^{*} \circ t^{*}(y)\right)\right) \\
& =\underbrace{t^{*} \circ \sigma^{*}}_{i d}\left(x \cdot t^{*}(y)\right)
\end{aligned}
$$

Algebraic 83 Geometric Topology, Volume 6 (2006) 


$$
=x \cdot t^{*}(y) .
$$

This completes the proof.

The usefulness of transfers in group cohomology lies to a large extent in the well known result, that for a group monomorphism $\gamma: G \rightarrow H$, the effect of the composition $t r_{\gamma}^{*} \circ \mathbb{B} \gamma^{*}$ in cohomology is simply multiplication by the index $[H: \gamma(G)]$. The situation for transfers between classifying spectra induced by fusion-preserving monomorphisms is similar. Although one might expect that the order of the outer automorphism groups of the fusion systems should come into play, it is only the order of the underlying $p$-groups that is important. The reason is that the characteristic idempotents, which are used to construct the transfer, have a "normalizing effect". In some sense they divide out the order of the outer automorphism groups.

Proposition 9.5 Let $\mathcal{F}_{1}$ and $\mathcal{F}_{2}$ be saturated fusion systems over $S_{1}$ and $S_{2}$, respectively, and let $\gamma: S_{1} \rightarrow S_{2}$ be a fusion-preserving monomorphism. Then the composition

$$
\operatorname{Tr}\left(\gamma_{\mathcal{F}_{1}}^{\mathcal{F}_{2}}\right)^{*} \circ \mathbb{B} \gamma_{\mathcal{F}_{1}}^{\mathcal{F}_{2}{ }^{*}}
$$

acts on $H^{*}\left(\mathbb{B} \mathcal{F}_{1}\right)$ as multiplication by $\left|S_{2}\right| /\left|S_{1}\right|$.

Proof Indeed,

$$
\begin{aligned}
\mathbb{B} \gamma_{\mathcal{F}_{1}}^{\mathcal{F}_{2}} \circ \operatorname{Tr}\left(\gamma_{\mathcal{F}_{1}}^{\mathcal{F}_{2}}\right) & =\sigma_{\mathcal{F}_{2}} \circ B \gamma \circ t_{\mathcal{F}_{1}} \circ \sigma_{\mathcal{F}_{1}} \circ t r_{\gamma} \circ t_{\mathcal{F}_{2}} \\
& =\sigma_{\mathcal{F}_{2}} \circ B \gamma \circ \tilde{\omega}_{\mathcal{F}_{1}} \circ t r_{\gamma} \circ t_{\mathcal{F}_{2}} \\
& \stackrel{(7.7)}{=} \sigma_{\mathcal{F}_{2}} \circ B \gamma \circ t r_{\gamma} \circ t_{\mathcal{F}_{2}} .
\end{aligned}
$$

Now, if $x \in H^{*}\left(\mathbb{B} \mathcal{F}_{1}\right)$, then

$$
\begin{aligned}
\operatorname{Tr}\left(\gamma_{\mathcal{F}_{1}}^{\mathcal{F}_{2}}\right)^{*} \circ \mathbb{B} \gamma_{\mathcal{F}_{1}}^{\mathcal{F}_{2}{ }^{*}}(x) & =t_{\mathcal{F}_{2}}^{*} \circ t r_{\gamma}^{*} \circ B \gamma^{*} \circ \sigma_{\mathcal{F}_{2}}^{*}(x) \\
& =t_{\mathcal{F}_{2}}^{*}\left(\frac{\left|S_{2}\right|}{\left|S_{1}\right|} \cdot \sigma_{\mathcal{F}_{2}}^{*}(x)\right) \\
& =\frac{\left|S_{2}\right|}{\left|S_{1}\right|} \cdot t_{\mathcal{F}_{2}}^{*}\left(\sigma_{\mathcal{F}_{2}}^{*}(x)\right) \\
& =\frac{\left|S_{2}\right|}{\left|S_{1}\right|} \cdot x
\end{aligned}
$$

This completes the proof.

Transfers between classifying spectra also exhibit Frobenius reciprocity.

Algebraic $8 \mathcal{G}$ Geometric Topology, Volume 6 (2006) 
Proposition 9.6 (Frobenius reciprocity) Let $\mathcal{F}_{1}$ and $\mathcal{F}_{2}$ be saturated fusion systems over $S_{1}$ and $S_{2}$, respectively, and let $\gamma: S_{1} \rightarrow S_{2}$ be a fusion-preserving monomorphism. If $x \in H^{*}\left(\mathbb{B} \mathcal{F}_{2}\right)$ and $y \in H^{*}\left(\mathbb{B} \mathcal{F}_{1}\right)$, then

$$
\operatorname{Tr}\left(\gamma_{\mathcal{F}_{1}}^{\mathcal{F}_{2}}\right)^{*}\left(\mathbb{B} \gamma_{\mathcal{F}_{1}}^{\mathcal{F}_{2}}(x) \cdot y\right)=x \cdot \operatorname{Tr}\left(\gamma_{\mathcal{F}_{1}}^{\mathcal{F}_{2}}\right)^{*}(y) .
$$

Proof Using the facts that $\sigma_{\mathcal{F}_{1}}^{*}$ is a ring homomorphism, and that $t_{\mathcal{F}_{2}}^{*}$ and $t r_{\gamma}^{*}$ both exhibit Frobenius reciprocity, we get

$$
\begin{aligned}
\operatorname{Tr}\left(\gamma_{\mathcal{F}_{1}}^{\mathcal{F}_{2}}\right)^{*}\left(\mathbb{B} \gamma_{\mathcal{F}_{1}}^{\mathcal{F}_{2}}(x) \cdot y\right) & =t_{\mathcal{F}_{2}}^{*} \circ t r_{\gamma}^{*} \circ \sigma_{\mathcal{F}_{1}}^{*}\left(\mathbb{B} \gamma_{\mathcal{F}_{1}}^{\mathcal{F}_{2}{ }^{*}}(x) \cdot y\right) \\
& =t_{\mathcal{F}_{2}}^{*} \circ t r_{\gamma}^{*} \circ\left(\left(\sigma_{\mathcal{F}_{1}}^{*} \circ \mathbb{B} \gamma_{\mathcal{F}_{1}}^{\mathcal{F}_{2}}(x)\right) \cdot \sigma_{\mathcal{F}_{1}}^{*}(y)\right) \\
& =t_{\mathcal{F}_{2}}^{*} \circ r_{\gamma}^{*}\left(\left(\mathbb{B} \gamma^{*} \circ \sigma_{\mathcal{F}_{2}}^{*}(x)\right) \cdot \sigma_{\mathcal{F}_{1}}^{*}(y)\right) \\
& =t_{\mathcal{F}_{2}}^{*}\left(\sigma_{\mathcal{F}_{2}}^{*}(x) \cdot\left(t r_{\gamma}^{*} \circ \sigma_{\mathcal{F}_{1}}^{*}(y)\right)\right) \\
& =x \cdot\left(t_{\mathcal{F}_{2}}^{*} \circ t r_{\gamma}^{*} \circ \sigma_{\mathcal{F}_{1}}^{*}(y)\right) \\
& =x \cdot \operatorname{Tr}\left(\gamma_{\mathcal{F}_{1}}^{\mathcal{F}_{2}}\right)^{*}(y) .
\end{aligned}
$$

This completes the proof.

The reciprocity results in this section can be shown to hold at the level of stable homotopy, as is the case with transfers induced by finite covers, when the structure maps preserve diagonals (in particular the classifying spectra involved must have diagonal maps). Since the only known cases where this happens is for fusion systems belonging to $p$-local finite groups, in which case the classifying spectra are suspension spectra, this discussion is postponed for [23].

\section{Comparison to stable classifying spaces}

We conclude the paper by comparing the theory of classifying spectra of saturated fusion systems with the theory obtained by infinite suspension of classifying spaces of finite groups and $p$-local finite groups, and proving that the theory of classifying spectra extends both these theories.

Algebraic 83 Geometric Topology, Volume 6 (2006) 
It is shown in [7] that when $\mathcal{F}$ has an associated centric linking system $\mathcal{L}$, the classifying spectrum $\mathbb{B} \mathcal{F}$ is homotopy equivalent to the $p$-completed suspension spectrum $\Sigma^{\infty}|\mathcal{L}|_{p}^{\wedge}$. We extend this observation to structured classifying spectra. The reader is referred to [7] for the precise definition of centric linking systems and $p$-local finite groups.

Proposition 10.1 Let $(S, \mathcal{F}, \mathcal{L})$ be a $p$-local finite group. Then the infinite suspension

$$
\Sigma^{\infty} \theta: \Sigma^{\infty} B S \longrightarrow \Sigma^{\infty}|\mathcal{L}|_{p}^{\wedge}
$$

of the natural inclusion $\theta: B S \rightarrow|\mathcal{L}|_{p}^{\wedge}$ is equivalent to the structure map

$$
\sigma_{\mathcal{F}}: \mathbb{B} S \longrightarrow \mathbb{B} \mathcal{F}
$$

Proof First we recall that $\mathbb{B} S$ is homotopy equivalent to $\Sigma^{\infty} B S$, and we may therefore identify the two via a chosen homotopy equivalence.

In [7] it is shown that $\theta$ is $\mathcal{F}$-stable and it follows that $\Sigma^{\infty} \theta$ is $\mathcal{F}$-stable. By Corollary 6.4 it follows that

$$
\Sigma^{\infty} \theta \circ \widetilde{\omega}_{\mathcal{F}} \simeq \Sigma^{\infty} \theta
$$

By construction of the structured classifying spectrum as a mapping telescope we get a map $h: \mathbb{B} \mathcal{F} \rightarrow \Sigma^{\infty}|\mathcal{L}|_{p}^{\wedge}$ such that

$$
h \circ \sigma_{\mathcal{F}} \simeq \Sigma^{\infty} \theta
$$

In particular the corresponding equality holds for the induced maps in cohomology with $\mathbb{F}_{p}$-coefficients. It is shown in [7] (see also Proposition 9.1) that in cohomology the maps $\sigma_{\mathcal{F}}$ and $\theta$ both induce injctions with image $H^{*}(\mathcal{F})$ in $H^{*}(B S)$, and therefore we conclude that $h$ induces an isomorphism

$$
h^{*}: H^{*}\left(\Sigma^{\infty}|\mathcal{L}|_{p}^{\wedge}\right) \stackrel{\cong}{\longrightarrow} H^{*}(\mathbb{B} \mathcal{F}) .
$$

Since the spectra involved are $p$-complete, we deduce that $h$ is a homotopy equivalence.

We now turn our attention to $p$-completed classifying spaces of finite groups. This theory overlaps in parts with the theory of classifying spaces of $p$-local finite groups since the classifying space of the $p$-local finite group induced by a finite group $G$ is homotopy equivalent to $B G_{p}^{\wedge}$. An additional aspect for the stable classifying spaces of groups is that the inclusion of a Sylow subgroup $S$ into a finite group $G$ has a stable transfer, which we compare with the transfer of a saturated fusion system.

Algebraic 6 Geometric Topology, Volume 6 (2006) 
Proposition 10.2 Let $G$ be a finite group with Sylow subgroup $S$. Then the map

$$
\mathbb{B} \iota_{S}: \mathbb{B} S \longrightarrow \mathbb{B} G
$$

induced by the inclusion $S \leq G$ is equivalent to the structure map

$$
\sigma_{\mathcal{F}_{S}(G)}: \mathbb{B} S \longrightarrow \mathbb{B} \mathcal{F}_{S}(G) \text {. }
$$

Furthermore, if we let $g$ be a homotopy inverse of the homotopy equivalence

$$
\mathbb{B} \iota_{S} \circ \operatorname{tr}_{S}: \mathbb{B} G \longrightarrow \mathbb{B} G
$$

then the map

$$
t^{\prime}:=t r_{S} \circ g: \mathbb{B} G \longrightarrow \mathbb{B} S
$$

is equivalent to the map

$$
t_{\mathcal{F}_{S}(G)}: \mathbb{B} \mathcal{F}_{S}(G) \longrightarrow \mathbb{B} S
$$

Proof Write $\mathcal{F}:=\mathcal{F}_{S}(G)$. It was shown in [7] that for a finite $p$-group $G$ with Sylow subgroup $S$, the map $B \iota_{S}: B S \longrightarrow B G$ is equivalent to the inclusion $\theta: B S \longrightarrow|\mathcal{L}|_{p}^{\wedge}$ of $B S$ into the classifying space of the corresponding $p$-local finite group. By Proposition 10.1 it follows that the map $\mathbb{B} \iota_{S}: \mathbb{B} S \longrightarrow \mathbb{B} G$ is equivalent to the map $\sigma_{\mathcal{F}}: \mathbb{B} S \longrightarrow \mathbb{B} \mathcal{F}$.

When proving the second claim we can fix a homotopy equivalence $\mathbb{B} \mathcal{F} \rightarrow \mathbb{B} G$ and regard $t^{\prime}$ as a map $\mathbb{B} \mathcal{F} \rightarrow \mathbb{B} S$. Assume, for now, that $\tilde{\omega}_{\mathcal{F}} \circ t r_{S} \simeq t r_{S}$. Then $\tilde{\omega}_{\mathcal{F}} \circ t^{\prime} \simeq t^{\prime}$ and we get

$$
t_{\mathcal{F}} \simeq t_{\mathcal{F}} \circ \mathbb{B} \iota_{S} \circ t^{\prime} \simeq t_{\mathcal{F}} \circ \sigma_{\mathcal{F}} \circ t^{\prime} \simeq \tilde{\omega}_{\mathcal{F}} \circ t^{\prime} \simeq t^{\prime}
$$

which is what we want to show.

To prove that $\omega_{\mathcal{F}} \circ t r_{S} \simeq t r_{S}$ it suffices, by Corollary 6.4, to establish that $t r_{S}$ is $\mathcal{F}$-stable, which is actually quite well known. One way to convince oneself of this is to note that $t r_{S}$ is the image in $\{B G, B S\}$ of $\left[S, i d_{S}\right] \in A(G, S)$, and that for $P \leq S$ and $\varphi \in \operatorname{Hom}_{\mathcal{F}}(P, S)$ one has

$$
\left[\varphi(P), \varphi^{-1}\right]_{S}^{P} \circ\left[S, i d_{S}\right]_{G}^{S}=\left[\varphi(P), \varphi^{-1}\right]_{G}^{P}=\left[P, i d_{P}\right]_{G}^{P} \in A(G, P),
$$

since $\varphi$ is a conjugation induced by an element of $G$.

With the notation of the preceding theorem, the stable characteristic idempotent of $\mathcal{F}_{S}(G)$ can be obtained as

$$
\tilde{\omega}_{\mathcal{F}_{S}(G)} \simeq t^{\prime} \circ \mathbb{B} \iota_{S},
$$

and the characteristic idempotent is then

$$
\omega_{\mathcal{F}_{S}(G)}=\tilde{\alpha}^{-1}\left(\tilde{\omega}_{\mathcal{F}_{S}(G)}\right),
$$

Algebraic 83 Geometric Topology, Volume 6 (2006) 
regarded as an element of $A(S, S)_{p}^{\wedge}$.

Another feature of the theory of $p$-completed classifying spaces of finite groups is that it is functorial. Namely, given a homomorphism $\bar{\gamma}: G_{1} \rightarrow G_{2}$ between finite groups, one gets a map of $p$-completed classifying spaces $B \bar{\gamma}_{p}^{\wedge}: B G_{1} \hat{p} \rightarrow B G_{2} \hat{p}$. Furthermore, the restriction $\gamma: S_{1} \rightarrow S_{2}$ to Sylow subgroups is fusion preserving for the fusion systems $\mathcal{F}_{S_{1}}\left(G_{1}\right)$ and $\mathcal{F}_{S_{2}}\left(G_{2}\right)$. Hence we get a map $\mathbb{B} \gamma_{\mathcal{F}_{1}}^{\mathcal{F}_{2}}$ of classifying spectra, which we can compare to $\mathbb{B} \bar{\gamma}$, the infinite suspension of $B \bar{\gamma}_{p}^{\wedge}$.

Proposition 10.3 Let $\bar{\gamma}: G_{1} \rightarrow G_{2}$ be a homomorphism of finite groups with a restriction $\gamma: S_{1} \rightarrow S_{2}$ to Sylow subgroups. Then the diagram

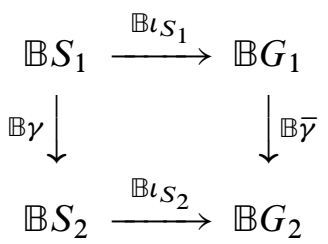

is equivalent to the diagram

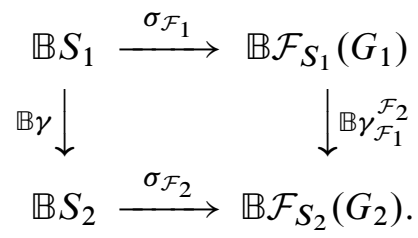

Proof By Proposition 10.2 we can replace all but the map on the right side of the upper diagram with the corresponding maps and objects on the lower diagram. All that remains is to show is now that, when thus regarded as a map $\mathbb{B} \mathcal{F}_{S_{1}}\left(G_{1}\right) \rightarrow \mathbb{B} \mathcal{F}_{S_{2}}\left(G_{2}\right)$, the map $\mathbb{B} \bar{\gamma}$ is homotopic to the map $\mathbb{B} \gamma_{\mathcal{F}_{1}}^{\mathcal{F}_{2}}$. Now, from the equivalence $\mathbb{B} \bar{\gamma} \circ \sigma_{\mathcal{F}_{1}} \simeq \sigma_{\mathcal{F}_{2}} \circ \mathbb{B} \gamma$ it follows that

$$
\mathbb{B} \bar{\gamma} \simeq \mathbb{B} \bar{\gamma} \circ \sigma_{\mathcal{F}_{1}} \circ t_{\mathcal{F}_{1}} \simeq \sigma_{\mathcal{F}_{2}} \circ \mathbb{B} \gamma \circ t_{\mathcal{F}_{1}}=\mathbb{B} \gamma_{\mathcal{F}_{1}}^{\mathcal{F}_{2}}
$$

\section{References}

[1] J F Adams, Stable homotopy and generalised homology, University of Chicago Press, Chicago, Ill. (1974) MR0402720

[2] J F Adams, Infinite loop spaces, Annals of Mathematics Studies 90, Princeton University Press, Princeton, N.J. (1978) MR505692

[3] J C Becker, D H Gottlieb, The transfer map and fiber bundles, Topology 14 (1975) 1-12 MR0377873

Algebraic 83 Geometric Topology, Volume 6 (2006) 
[4] D J Benson, M Feshbach, Stable splittings of classifying spaces of finite groups, Topology 31 (1992) 157-176 MR1153243

[5] A K Bousfield, D M Kan, Homotopy limits, completions and localizations, Springer, Berlin (1972) MR0365573

[6] C Broto, R Levi, B Oliver, Homotopy equivalences of p-completed classifying spaces of finite groups, Invent. Math. 151 (2003) 611-664 MR1961340

[7] C Broto, R Levi, B Oliver, The homotopy theory of fusion systems, J. Amer. Math. Soc. 16 (2003) 779-856 MR1992826

[8] G Carlsson, Equivariant stable homotopy and Segal's Burnside ring conjecture, Ann. of Math. (2) 120 (1984) 189-224 MR763905

[9] N Castellana, L Morales, Vector bundles over classifying spaces revisited, in preparation

[10] W G Dwyer, Transfer maps for fibrations, Math. Proc. Cambridge Philos. Soc. 120 (1996) 221-235 MR1384465

[11] R Kessar, R Stancu, A reduction theorem for fusion systems of blocks, preprint

[12] L G Lewis, J P May, J E McClure, Classifying G-spaces and the Segal conjecture, from: "Current trends in algebraic topology, Part 2 (London, Ont., 1981)", CMS Conf. Proc. 2, Amer. Math. Soc., Providence, R.I. (1982) 165-179 MR686144

[13] M Linckelmann, P Webb, unpublished work

[14] J Martino, S Priddy, Stable homotopy classification of $B G_{p}^{\wedge}$, Topology 34 (1995) 633-649 MR1341812

[15] J Martino, S Priddy, Unstable homotopy classification of $B G_{p}^{\wedge}$, Math. Proc. Cambridge Philos. Soc. 119 (1996) 119-137 MR1356164

[16] J P May, J E McClure, A reduction of the Segal conjecture, from: "Current trends in algebraic topology, Part 2 (London, Ont., 1981)", CMS Conf. Proc. 2, Amer. Math. Soc., Providence, R.I. (1982) 209-222 MR686147

[17] G Nishida, Stable homotopy type of classifying spaces of finite groups, from: "Algebraic and topological theories (Kinosaki, 1984)”, Kinokuniya, Tokyo (1986) 391-404 MR1102269

[18] B Oliver, Equivalences of classifying spaces completed at the prime two, Mem. Amer. Math. Soc. (to appear)

[19] B Oliver, Equivalences of classifying spaces completed at odd primes, Math. Proc. Cambridge Philos. Soc. 137 (2004) 321-347 MR2092063

[20] L Puig, Frobenius systems and their localizing categories, preprint

[21] L Puig, unpublished notes

[22] K Ragnarsson, Alternative stable homotopy classification of $B G_{p}^{\wedge}$, Topology (to appear) 
[23] K Ragnarsson, Retractive transfers and p-local finite groups, preprint

[24] K Ragnarsson, A Segal conjecture for p-completed classifying spaces, preprint

[25] T tom Dieck, Transformation groups and representation theory, Lecture Notes in Mathematics 766, Springer, Berlin (1979) MR551743

Department of Mathematical Sciences, University of Aberdeen

Aberdeen AB24 3UE, UK

kari@maths.abdn.ac.uk

Received: 25 March 2005 Revised: 19 January 2006 